\title{
Discutendo con Christof Dipper
}

\section{Interventi di Paolo Macry, Fulvio Cammarano, Vinzia Fiorino, Antonio Bonatesta e Andrea Claudi}

\begin{abstract}
Discutendo con l'analisi di Christof Dipper, Paolo Macry osserva che - se non poche sue osservazioni sono fondate - allo storico tedesco sfugge il carattere in movimento della storiografia contemporaneistica italiana. Fulvio Cammarano aggiunge che alcune questioni di metodo inficiano le analisi di Dipper, a partire dall'assenza di una precisa analisi quantitativa. Vinzia Fiorino, invece, rileva la fondatezza di varie critiche avanzate da Dipper, e rileva non pochi ritardi nella storiografia italiana, a partire da quelli nel campo della storia culturale (concorda, fra l'altro, nel rilevare un notevole squilibrio di genere nel corpo docente). Infine Antonio Bonatesta e Andrea Claudi argomentano che molti dei problemi della storiografia contemporaneistica italiana dipendono da questioni strutturali generali, politiche ed economiche, e propongono una prima analisi quantitativa del corpo docente attuale della contemporaneistica italiana.
\end{abstract}

Parole chiave: Storiografia, Storia contemporanea, Abilitazione scientifica nazionale (Asn)

\section{Paolo Macry, Fulvio Cammarano, Vinzia Fiorino, Antonio Bonatesta, Andrea Claudi,} A Debate with Christof Dipper

Paolo Macry engages with Chrisof Dipper's argument and points out that, despite the validity of that many of his points, the German historian misses the originality that characterizes contemporary Italian historiography. Fulvio Cammarano adds that Dipper's essay is methodologically flawed, and lacks a precise quantitative analysis. Vinzia Fiorino, on the other hand, highlights the merits of some of Dipper's critiques, and argues that Italian historiography is in many ways behind, for instance in the field of cultural history (and she agrees with Dipper that there is a strong gender inequality among faculty members). Finally, Antonio Bonatesta and Andrea Claudi point out that many of the problems of contemporary Italian historiography depend on more general structural, political and economic issues, and argue that what is needed is a thorough quantitative study of university faculties.

Key words: Historiography, Contemporary History, Research Assessment 


\section{Secche e sviluppi}

\section{Paolo Macry*}

Christof Dipper è sperimentato conoscitore della storiografia italiana, ha lavorato anche su temi di storia italiana, ha presieduto la Arbeitsgemeinschaft für die Neueste Geschichte Italiens, condividendone l'intento di costituire un ponte tra cultura italiana e cultura tedesca. La sua non è una firma qualunque. E anche i suoi giudizi non passano inosservati. Tanto più sembra opportuno discutere sia pur brevemente le pagine che, in un articolo comparso su "Vierteljahrshefte für Zeitgeschichte", lo studioso ha voluto dedicare a La storia contemporanea in Italia (così recita il titolo tedesco).

Dipper inizia con una descrizione molto critica del nostro sistema universitario. Lo giudica, nel quadro europeo, particolarmente subalterno ai "giochi della politica". Ritiene che il reclutamento sia avvenuto spesso senza adeguati "criteri di efficienza", riferendosi non soltanto alle immissioni in ruolo del 1967 e del 1973, ma anche alla successiva gestione delle assunzioni da parte degli atenei, a seguito delle riforme di Antonio Ruberti e poi di Luigi Berlinguer. "Nel Paese del familismo e del clientelismo", annota al proposito, il riconoscimento dell'autonomia universitaria non poteva che provocare danni. La conseguenza delle "promozioni di massa" deliberate da governi e parlamenti e coltivate dalle sedi universitarie sarà l'affermarsi di un corpo docente ipertrofico, localistico e vecchio. Oggi gli storici accademici sono più numerosi in Italia che in Germania, mentre "una parte considerevole dei ricercatori è costituita da sessantenni senza ulteriori titoli oltre la laurea, ma in compenso con lo stesso carico d'insegnamento dei professori ordinari', osserva Dipper. La didattica gli appare molto carente, seminari ed esercitazioni sono merce rara, le infrastrutture insufficienti, numerosi i docenti di ruolo che fanno altri lavori. Nelle università italiane, conclude con parole draconiane, gli studenti "ricevono un insegnamento accademico di norma pessimo, che comprende ben poco oltre i corsi ordinari, in cui professori o ricercatori recitano dai loro libri di testo". Un circolo vizioso, che viene difeso da chi ne fa parte organica e protetto da sindacati i quali uniscono alla "mentalità assistenziale" il rifiuto dell'idea di competizione, sostituita con "la parola d'ordine della lotta di classe".

Si tratta di rilievi che hanno più di un fondo di verità. L'inadeguatezza e il corporativismo con cui le autorità ministeriali e il corpo accademico hanno talvolta risposto alle tensioni e trasformazioni (culturali e strutturali) del secondo Novecento - e anzitutto alla cosiddetta "università di massa" - sono sotto gli occhi di tutti. La produttività scientifica e l'efficacia didattica ne hanno risentito.

* Università degli studi di Napoli Federico II; paolo.macry@unina.it 
Ma stupisce, nonostante ciò, che uno studioso equilibrato come Dipper finisca per cadere in generalizzazioni ed esagerazioni le quali rischiano di inficiare la credibilità del giudizio. Appare poco ragionevole una lettura uniformemente e radicalmente critica di cicli politici che vanno dalla liberalizzazione degli accessi universitari e dei piani di studio (1969) fino ai provvedimenti del ministro Mariastella Gelmini (2008-2011). Di mezzo, dopotutto, ci sono decenni. Distinguere sarebbe doveroso. E non è un caso che, a questo punto, la chiave di spiegazione diventi "il paese del familismo e del clientelismo", ovvero il più classico dei (supposti) caratteri originari degli italiani. Uno di quegli stigmi che, volendo spiegare tutto, rischiano di non spiegare nulla. Dipper, peraltro, non dovrebbe ignorare le disparità talvolta vistose che esistono - non certo da oggi, ma ancora oggi — tra ateneo e ateneo, le modalità non così uniformi nella prassi del reclutamento, la diversa produttività didattica dei corsi di laurea, le carriere non omogenee dei laureati e dei dottori di ricerca.

È però nel giudizio sulla storiografia dell'età contemporanea che l'articolo dello studioso tedesco appare particolarmente discutibile. Dipper illustra lo stato dell'arte della disciplina sulla base dell'esperienza fatta come membro della Commissione per l'abilitazione all'insegnamento della Storia contemporanea (anni 2012/2013 e 2013/2014). Fa riferimento cioè a quegli oltre seicento candidati all'abilitazione di prima e seconda fascia che è stato suo compito vagliare in sede di commissione e che lui stesso descrive come "l'insieme delle nuove leve accademiche". Un numero cospicuo che, depurato da alcune decine di freelance presumibilmente estranei all'accademia e alla ricerca (giornalisti, insegnanti di scuola, impiegati pubblici ecc.), sembrerebbe comunque un buon punto di osservazione per cogliere i caratteri della storiografia contemporaneistica italiana. O meglio i caratteri di chi si ritiene in grado di aspirare al ruolo di professore universitario, cioè non della più giovane storiografia. Il problema, però, è che Dipper utilizza un campione corrispondente al 10 per cento del totale dei candidati: una cinquantina di studiosi, i cui criteri di selezione non vengono peraltro spiegati al lettore. Il procedimento è corretto? Il campione è significativo? Fatto sta che, non diversamente dall'analisi del sistema universitario, il giudizio sugli studi appare fin troppo netto. Con una singolare mescolanza tra descrizioni lucide e generalizzazioni, rilievi acuti e luoghi comuni.

Quali sono, secondo Dipper, le criticità della contemporaneistica italiana? In primo luogo, una selezione gravemente manchevole della cronologia, che, per fare l'esempio più vistoso, finisce per espungere dagli studi proprio quell'“Ottocento lungo" - dall'età napoleonica al 1915 - che è la stagione decisiva per la nascita della coscienza nazionale del Paese. Sorprendentemente, dice l'accademico tedesco, "il Risorgimento, con poche eccezioni, scompare dal campo visivo degli studiosi", mentre "il XIX secolo sul piano della storia dello Stato non trova più alcuno spazio" e "lo stesso vale per la prima guerra mondiale, i cui studiosi si contano sulle dita di una mano". All'altro capo della periodizzazione contemporaneistica, del resto, la ricerca si ferma "con poche eccezioni" 
agli anni Settanta del Novecento, sicché "manca del tutto un qualsiasi sguardo storiografico a sostegno di un'analisi del tempo presente". Per sapere qualcosa del fenomeno Berlusconi, esemplifica Dipper, bisogna ricorrere agli scritti di Paul Ginsborg. In secondo luogo, gli studi mostrano "una forte tendenza al localismo" e "un alto grado di autoreferenzialità". Un fenomeno, questo, che Dipper collega alla crisi ormai pluridecennale dello Stato centrale. Il che spiegherebbe perché in particolare nel Mezzogiorno sia oggi possibile parlare di "un boom della ricerca localistica", ma anche perché esistano in Italia "storici al servizio della chiesa, i quali [...] producono da devoti per i devoti", o storici che si dedicano in modo autoreferenziale ai partiti e sindacati ai quali sono essi stessi vicini. In terzo luogo, raramente i ricercatori italiani sembrano occuparsi di altro che del proprio paese. Vittime di un "narcisismo nazionale" che appare senza limiti. "É sorprendente", scrive Dipper, "che nel campione degli aspiranti alla cattedra di ordinario solo in tre forniscano contributi alla storia extraitaliana".

Per il resto, l'accademico tedesco deplora il descrittivismo degli studi, la mancanza di un adeguato approccio teorico o quanto meno problematico, il conformismo nella scelta dei temi di ricerca ("troppo pericoloso", soprattutto per i giovani, avventurarsi su strade nuove), la preponderanza di un taglio politico-evenemenziale ("una forma di racconto oggettivo della storia politica"), il "persistente strapotere della tradizione di Benedetto Croce e Antonio Gramsci", l'influenza del cattolicesimo, la debolezza della storia sociale. E poi l'italica predilezione per il bel scrivere ("la gioia di raccontare"), una qualche scoria erudita, la poca conoscenza delle lingue straniere ecc. Inutile aggiungere, se tutto questo è vero, che "la massa degli storici contemporaneisti non è per nulla innovativa".

Il quadro a tinte fosche viene poi costellato da ulteriori spigolature che non si capisce se vogliano essere più severe o più ironiche. Dei ricercatori italiani, Dipper snocciola una quantità di manchevolezze, furbizie, malvezzi che di certo non cospirano alla loro gloria. Abituati a scrivere troppo, sono sempre alla ricerca di un qualsivoglia editore e concorrono a improbabili premi che possono fruttare qualche finanziamento, ma li obbligano a occuparsi dei temi specifici sui quali è bandito il premio. Intrattengono perciò rapporti con comuni, province, regioni, banche, cooperative, fondazioni. Peccati veniali o peccati capaci di pregiudicare la ricerca, verrebbe da chiedersi? Quegli stessi studiosi preferiscono non di rado pubblicare i propri articoli su riviste che mancano di peer review, ma funzionano come benevoli "punti d'incontro di amici e confratelli". Inoltre, appaiono troppo disinvolti circa le regole del mestiere, spesso omettendo nei loro libri ogni rassegna degli studi, allegando "raramente" una bibliografia al testo, dimenticando talvolta di illustrare le fonti utilizzate. In compenso, annota Dipper, non manca mai "un indice dei nomi, grazie al quale amici, aiutanti e santi protettori possono sapere con uno sguardo quante volte sono stati citati'. Come se l'uso improprio di questo strumento (un altro peccato veniale, direi) ne eliminasse l'importanza. 
Una requisitoria con tutti i crismi, come si vede. Che tuttavia lascia in sospeso la domanda delle domande: di cosa stiamo parlando? Della contemporaneistica italiana in questo scorcio di terzo millennio? Oppure dei cinque-seicento aspiranti nel 2012-2014 all'abilitazione universitaria? O invece della cinquantina di studiosi selezionati dallo stesso Dipper? Se si cerca qualche verifica quantitativa ai suoi giudizi, ad esempio prendendo in esame gli oltre 450 volumi recensiti dal benemerito "Il mestiere di storico" tra quelli pubblicati nel 2013 (si vedano i due fascicoli editi l'anno successivo, come di consueto), il quadro appare, almeno in parte, diverso. Certo, anche questa è una selezione della contemporaneistica - sono i volumi che i redattori del "Mestiere" hanno ritenuto degni di attenzione - e dunque ne fornisce un panorama parziale. Ne rappresenta, in qualche modo, il meglio. E tuttavia, trattandosi di centinaia di casi, il campione qualcosa dice.

Ebbene, tra i volumi presi in esame nel 2014, molti sono effettivamente dedicati alla storia politica (e tuttavia, accanto alle ricostruzioni evenemenziali, non mancano le storie della politica, dei modelli politici, dei sistemi amministrativi, dello Stato). Molti sono, per altro verso, i saggi di storia strutturale, dell'economia, del lavoro, dell'impresa, del consumo, dell'ambiente: talvolta anch'essi tradizionali, altre volte tesi all'utilizzo di categorie innovative. E molti, tornando al problema della periodizzazione, sono gli studi sul Risorgimento, probabilmente il settore storiografico che ha fatto registrare i tentativi più incisivi di rilettura analitica e interpretativa della vicenda nazionale e statuale del Paese. Del nuovo Risorgimento culturalista inaugurato ormai diversi anni fa da Alberto Banti si può pensare tutto il bene possibile o invece avere i propri dubbi, ma è un fatto che quello degli ottocentisti è ormai un esercito (un esercito assai diverso dalla tradizione delle "storie patrie"). E basti citare i molti studiosi che hanno contribuito al volume degli Annali della Storia d'Italia Einaudi su $\mathrm{Il}$ Risorgimento (a cura di A.M. Banti e Paul Ginsborg) o, in un'ottica diversa da quella bantiana, gli innumerevoli storici - anche giovani - chiamati da Mario Isnenghi a comporre i sette grossi tomi degli Italiani in guerra editi dalla Utet. Che nel campione di Dipper (diversamente dal campione del "Mestiere") tutto questo scompaia è un segno della sua debole rappresentatività. Ma neppure sembra vero che la storiografia contemporaneistica si concentri sul periodo fascista e sia restia ad affrontare i temi dell'Italia repubblicana. Anche in questo caso, al di là dei molti (forse troppi) saggi di sintesi sui settant'anni del dopoguerra, non è possibile ignorare gli studi recenti dedicati, per esempio, alla crisi del sistema politico negli anni Novanta e alla stagione della nuova destra. O i molti ricercatori che, su riviste come "Meridiana", portano avanti l'analisi e la riflessione su un Sud otto-novecentesco riletto, con una certa vivacità, in chiave politico-culturale.

Quanto alla dimensione territorialmente limitata alla penisola della storiografia italiana, Dipper mette senza dubbio il dito nella piaga. È un fatto che, a lungo, gli studi hanno evitato una chiave analitica ed ermeneutica a scala com- 
parativa - europea o extraeuropea - che avrebbe potuto irrobustire e rinnovare la lettura del caso italiano, inserendolo proficuamente nelle discussioni e tematiche internazionali. Esempi come il dibattito agitato a metà Novecento da Rosario Romeo sul Risorgimento e il nodo storico-teorico del capitalismo industriale restano un'eccezione. E tuttavia, anche su questo piano, non sembra mancare qualche significativa novità, come gli studi ottocentisti sulla dimensione europea del liberalismo italiano, sul fenomeno continentale dell'esilio politico, sulle relazioni politico-ideologiche tra la penisola e l'America Latina ecc. $\mathrm{O}$ le ricerche, più numerose che nel passato e talvolta significative, dedicate alla Francia e alla Gran Bretagna, all'Europa di lingua tedesca e all'Europa orientale, al Mediterraneo e all'Africa coloniale e post-coloniale, alle Americhe. Si tratta di tendenze germinali o addirittura di casi singoli ed è comprensibile perciò che possano sfuggire a un campione molto selezionato come quello "concorsuale" di Dipper (che naturalmente non include gli studiosi collocati al di fuori del settore scientifico-disciplinare M-sto/04). E tuttavia l'eco di questa saggistica esplicitamente internazionalizzata e il prestigio acquistato dai suoi autori segnala un interesse, da parte della comunità scientifica, che forse non va sottovalutato. Fa pensare ai semi che germogliano. E altri semi che germogliano sono senza dubbio gli ormai molti convegni e seminari organizzati dalla Società italiana per lo studio della storia contemporanea (Sissco), al cui interno, soltanto a scorrerne i temi, i relatori e i discussant, sembrano rompersi molte delle vecchie consuetudini di ricerca e dei tradizionali comparti accademici (il che, sia detto tra parentesi, può anche destare qualche apprensione in chi, avendo una certa età, è cresciuto nel clima ben altrimenti strutturato - nel bene e nel male — delle "scuole" di secondo Novecento).

Anche questi pochi e parzialissimi cenni sembrano comunque confermare il dubbio che la casistica utilizzata da Dipper nelle sue pagine sia quantitativamente limitata, abbastanza fortuita e dunque distorsiva della realtà storiografica in questione. Né basta che l'autore renda esplicita la fonte dei propri giudizi, perché poi è lo stesso Dipper a suggerire - persuasivamente, retoricamente - che quella che descrive sia tout court la situazione della contemporaneistica italiana. Che cioè la parte valga per il tutto. Lo studioso tedesco esprime giudizi consolidati e dunque, come capita ai giudizi consolidati e agli stessi luoghi comuni, non privi di argomenti e in parte condivisibili. Ma (questo è il punto) solo in parte. Nelle sue opinioni tranchant spunta non di rado la coda del diavolo della verità. Come quando parla di descrittivismo, ipertrofia dell'evenemenziale, localismo ecc. È difficile non riconoscere che esista una fetta della contemporaneistica la quale risulta attardata in temi, metodi e interpretazioni di qualità mediocre. Ma si tratta di giudizi che andrebbero collocati nel tempo, aggiornati sulla base delle tendenze emergenti, ponderati tra i due poli della tradizione e dell'innovazione. Storicizzati insomma. Le generalizzazioni - troppe, talvolta troppo facili - depotenziano invece il ragionamento. Finiscono per portare Dipper sulle secche dello stereotipo. Gli impediscono di co- 
gliere taluni sviluppi in atto, che rendono questo campo di studi più promettente di quanto non suggerisca il suo quadro.

\section{Questioni di metodo}

\section{Fulvio Cammarano*}

Christof Dipper è uno stimato storico tedesco che conosce per lunga frequentazione l'Italia e diversi ambienti della storiografia italiana. Ha fatto parte della Commissione per l'Abilitazione scientifica nazionale (Asn) del 2012-2013 per il settore M-sto/04. Le pagine che qui discutiamo nascono proprio dall'esperienza di quei mesi. Se Dipper si fosse limitato a presentare il "diario" della sua attività di commissario, avremmo potuto leggere un'utile riflessione sui concreti problemi incontrati da un storico non italiano in occasione di quella valutazione, aneddoti e curiosità comprese. In realtà, Dipper ha scelto di andare ben oltre la cronaca, tra l'altro originariamente destinata ai lettori tedeschi, di un'esperienza molto peculiare, per proporre un giudizio complessivo sulla storia contemporanea in Italia. Ha cercato cioè di trasformare la sua "istantanea" concorsuale in un "film" - con tutti i problemi che s'incontrano nel passare da un genere ad un altro - "film" che peraltro è ora meritoriamente proposto di "proiettare" anche in Italia. Per fare questo tipo di operazione si è però dovuto avventurare in una rapida disamina della recente storia dell'Università italiana e del suo funzionamento oltre che nella sommaria descrizione degli usi e costumi dell'accademia italiana, prima di planare sulla questione da cui era partito, quella dei contenuti dei lavori presentati dai candidati all'Asn.

Si tratta di una sintetica cavalcata il cui scopo, il lettore lo capisce subito, è soprattutto quello di ribadire problemi e carenze del nostro sistema universitario e i limiti dell'approccio scientifico della storiografia contemporaneistica. Gli esempi riportati sembrano infatti avere il compito di confermare un immaginario già radicato nella vulgata nazionale e internazionale: la storia contemporanea italiana è, con rare eccezioni per lo più ascrivibili ad alcuni amici studiosi della Germania, una desolante landa di familismo e immoralità attraversata da ricerche povere e di scarso appeal. Si badi bene! Quasi nulla di quello che è scritto nell'intervento di Dipper ci giunge nuovo o può essere considerato avulso dalla realtà. Più volte all'interno della Società italiana per lo studio della storia contemporanea (Sissco) e dei comitati scientifici dei dottorati in storia sono emerse lamentele su limiti e problemi di alcuni filoni di ricerca nella storio-

\footnotetext{
* Università degli studi di Bologna; fulvio.cammarano@unibo.it
} 
grafia nazionale. Diverse affermazioni risultano quindi plausibili e non sarebbe difficile per chiunque sia dell'ambiente trovare qualche esempio in grado di confermarle, ma il vero problema è che il saggio intende dar conto dello stato complessivo della storia contemporanea e quindi, per questo tipo di obiettivo, non è accettabile in quanto del tutto carente per metodo e documentazione che appare decisamente inadeguata alla vastità delle tesi riportate. A dire il vero l'intero testo è un collage impressionistico in cui sembra mancare persino la voglia di dimostrare, documentare, uscire dal generico, portare dati e bibliografie solide (molte fonti sembrano rapsodiche: per l'analisi della "decennale eutanasia" del sistema universitario italiano si rinvia ad un solo articolo su un periodico online). Quasi tutto quello che si legge soffre d'imprecisione (una tra tante: in Italia il primo ciclo di dottorato non è del 1980 - anno in cui si approva la legge - ma del 1985), appare confezionato con dati approssimativi, mentre non pochi giudizi risultano francamente apodittici. Passiamone in rassegna qualcuno avendo ben chiaro che è l'impianto complessivo a non funzionare. Conosciamo tutti il pericolo della profezia che si autoavvera, in questo caso si può parlare della tesi che si auto conferma grazie a pochi, facili, esempi.

Gli studenti universitari "ricevono un insegnamento accademico di norma pessimo (...) in cui professori o ricercatori recitano (?) dai loro libri di testo. L'insegnamento basato sulla ricerca è la grande eccezione". Inutile per i lettori cercare riferimenti a queste sentenze. Personalmente, per esempio, non conosco colleghi che recitano i loro libri a lezione e che non inseriscano almeno parti della loro ricerca nella docenza. Sono però consapevole di riferirmi ad un campione probabilmente poco significativo e per questo evito di scrivere articoli sull'argomento. Ma Dipper, che l'articolo lo ha scritto, come può pensare di cavarsela con quegli inutili "di norma" o "eccezione"? Confermare l'esistenza di quella tipologia di professori richiederebbe in primo luogo riportare qualche cifra, avviare una campionatura per comprendere il loro effettivo spazio accademico e quindi dare conto - anche con dati solo orientativi, dato l'argomento - dell'incidenza di quel "gruppo" rispetto a quello dei docenti che si comportano diversamente.

La musica non cambia anche quando si entra in modo più specifico nel tema dell'Asn. Il problema principale è che Dipper nonostante sia uno storico di grande esperienza ha assunto quel campione di candidati come se fosse un campione statistico casuale e non come il frutto di una sedimentazione "storica" che ha finito per lasciare traccia sia sui profili curriculari, sia sulle tipologie dei lavori presentati. L'autore parla di poca professionalità tra le fila degli aspiranti all'abilitazione, costruisce anche un'utile tabella per "impieghi e attività", ma tale operazione non ha una riflessione critica alla base. I dilettanti non possono essere individuati solo sulla base di situazioni professionali magari momentanee e debbono essere distinti dai professionisti solo dalla qualità delle loro ricerche e non per la professione dichiarata che talvolta è solo l'esito di vicende biografiche e casualità della vita. Anche una riflessione (sociologica) sul- 
la genealogia dei temi e degli approcci, su cui Dipper s'interroga in modo talvolta ingenuo, non può prescindere da un simile spaccato.

Le difficoltà metodologiche palesate da questo lavoro sono inoltre amplificate dalla eccessiva mobilità del campione preso in esame. Alcune volte i testi presentati dai candidati in quel concorso sembrano offrirsi come riferimento esaustivo di un'intera produzione nazionale, ma a tratti compaiono qua e là, per di più a scopo di conferma negativa, professori ordinari e altre figure che con il concorso non c'entrano nulla. Paul Ginsborg - definito come l'unico ad avere analizzato il fenomeno Berlusconi (qui sarebbe bastato anche solo il veloce utilizzo di un motore di ricerca per evitare un simile errore) - o Emilio Gentile e Alberto Banti. Inserimenti random che aumentano l'incertezza sul materiale preso in considerazione da Dipper per la sua descrizione della storiografia in Italia. Sbalorditiva l'affermazione che la nostra storia politica non è altro che "l'analisi di partiti decaduti o di uomini politici morti da tempo". Non posso esimermi, dato l'ambito, di ricordare una cosa ben nota agli storici della politica non solo italiani, cioè che dal 1980 il gruppo di lavoro di cui faccio parte, fondato da Paolo Pombeni, ha avviato un lavoro di ripensamento delle fondamenta della storia politica proprio a partire dalla critica a quella storiografia agiografica e di corte. Su quanto si sia da allora innovata non sta a me dirlo, sul fatto che neppure compaia nei radar di chi scrive di storiografia in Italia è questione che lascio alle considerazioni di chi conosce la storiografia italiana. Anche l'elevata produttività degli storici italiani se confrontata con quella dei colleghi tedeschi non appare per l'autore occasione di riflessione sulle condizioni materiali in cui si trovano a operare i giovani studiosi e sui percorsi obbligati da attraversare per entrare nel sistema universitario, ma è una questione che si ferma sulla soglia della tradizione culturale: agli italiani piace raccontare, scrive il collega tedesco, e quindi sono rare le opere impostate analiticamente, così come le considerazioni teoriche poste alla base delle ricerche. Va da sé, quindi, che per Dipper l'italica "gioia di raccontare" spesso non "dispone di bibliografie, il che rende ancora più difficile il materiale citato; anche riportare dettagliatamente le fonti originali non costituisce la norma. Al contrario raramente manca un indice dei nomi, grazie al quale amici, aiutanti e santi protettori possono sapere con uno sguardo quante volte sono stati citati”. In realtà proprio il collega tedesco c'impedisce di accedere a fonti e bibliografie del suo ragionamento dato che mancano dati, statistiche, che ci facciano capire la misura di un simile disastro. Inoltre andrebbe considerato che non pochi colleghi considerano gli apparati bibliografici separati dalle note degli esercizi di virtuosismo informatico e quindi inutili, mentre a chi con il libro ci lavora, l'indice dei nomi in testi specialistici fornisce uno strumento importante al di là della rapidità di vedere l'eventuale citazione. Per Dipper, poi, "molte riviste sono ancora, come da prassi nel XIX secolo, punto d'incontro di amici e confratelli. Non pochi di questi periodici si collocano al di fuori del mondo scientifico, ma chiaramente non al di fuori di quello accademico". Tutto sembra ruotare esclusivamente nel 
mare magnum della collusione e del familismo: "quasi ogni libro ha una premessa o un'introduzione scritta da terzi", a scopo, inutile dirlo, clientelare. Non si salvano le tesi di dottorato: "senza prefazione del relatore sono una grande eccezione". Da qualche tempo, forse non a caso, alcuni colleghi e il sottoscritto hanno lanciato la "campagna" per spingere la storiografia a "dare i numeri" su ciò di cui parla, almeno ogniqualvolta questi siano reperibili. Pertanto chiedo a Dipper di aderire al nostro invito e riscrivere l'articolo corredandolo di alcuni elenchi, ma anche di alcune categorie interpretative che ci permettano di dire quante riviste sono "congreghe" di confratelli, quante collane editoriali sono sinonimo di malaffare, quanti premi sono una finzione. A proposito di categorie l'autore, questa volta parlando dell'intera storiografia e non solo della produzione dei candidati (un'alternanza continua e decisamente problematica per il lettore), cerca di dimostrare, sempre senza dati, che gli storici italiani raramente sentono la "necessità di fornire motivazioni teoriche per la scelta dei temi e per l'impalcatura della ricerca". In Germania, a differenza che in Italia, "nessuno storico tedesco può espressamente pensare che basti scrivere pragmaticamente a braccio". Il fatto è che non è chiaro cosa per Dipper sia concettuale, a parte l'approccio di Koselleck alla "storia dei concetti". Sembra infatti ignorare il discorso weberiano sui tipi ideali, sulle "regolarità della politica", sui meccanismi di legittimazione/delegittimazione. Forse ricordare che pochi anni fa il finanziamento Prin è stato assegnato a una ricerca (a cui hanno partecipato sette atenei) sulle declinazioni storiche della categoria di delegittimazione, categoria interpretativa che diversi studiosi tedeschi, inglesi e francesi hanno ritenuto introdotta nell'indagine storiografica proprio dagli storici italiani potrebbe essere evocativo di un clima culturale diverso da quello staticissimo in cui il collega tedesco ci vede immersi, in cui tutti o quasi tutti gli storici italiani scrivono senza troppo consapevolezza interpretativa "pragmaticamente a braccio". È un solo esempio e molti altri se ne potrebbero fare sulla base delle molte categorie interpretative con cui si passano in rassegna il XIX e XX secolo in chiave spesso comparata.

Inoltre è opportuno aggiungere che il valore attribuito da Dipper alla "storia comprendente" fa parte della sua cultura, e non potrebbe essere altrimenti per un allievo di Koselleck. Però esistono anche altri approcci, compreso quello della storia narrativa, a cui si riconoscono molti meriti. Come sempre sono le domande a fare la storia e non le prescrizioni che a turno vengono fornite da chi ritiene il proprio "sistema" migliore. Affermazioni poco accurate vengono poi fatte quando si affronta il tema dell'internazionalizzazione della storiografia italiana. Qui si torna al campione dei candidati all'Asn. "È sorprendente che nel campione degli aspiranti alla cattedra di ordinario solo in tre forniscano contributi alla storia extraitaliana (...). Un'interpretazione razionale di questa circostanza non è possibile, dal momento che si tratta apertamente di risultati fortuiti (...) ma non può trattarsi solo di una coincidenza. Dipende dall'età?'. Solo in parte e comunque è evidente che oggi nelle giovani generazioni c'è molta più 
apertura e comparazione di quella che si poteva trovare trent'anni fa. Dipper ha misurato il rilievo di questi dati sugli aspiranti ordinari, ma probabilmente è un campione poco rappresentativo. Avrebbe avuto più senso misurarlo sugli aspiranti associati. Inoltre, andrebbe ricordato che per la storia dei Paesi non europei la ragione principale di tale mancanza consiste semplicemente nel fatto che quegli studi ci sono, ma sono stati sottoposti a un'altra commissione, quella di Storia delle relazioni internazionali e delle aree extra-europee.

Sono molti altri i punti dell'articolo che mantengono questo carattere impressionistico. Non è possibile ovviamente seguire l'autore sulla strada della comparazione dei sistemi e del funzionamento delle macchine universitarie italiana e tedesca. Si tratta infatti di una comparazione che non si misura mai in termini di politiche e di finanziamenti di sistema e quindi anch'essa occasionale e finalizzata a una tesi preconcetta mai sottoposta alla concreta verifica dei dati. Né ci si può soffermare sulla complicata questione del localismo degli studi che al di là della consistenza effettiva Dipper non mette mai in connessione con il carattere nazionale del concorso in cui tutti i lavori confluiscono in un'unica commissione, a differenza del sistema tedesco dove molti di quegli studi non hanno modo di fuoriuscire dalle sedi locali, a causa del tipo di concorso regionale.

Inutile seguire ogni tema perché il problema principale rimane sempre quello di tipo metodologico, e così si rischia solo di ripetersi. In ambito scientifico non si può discutere criticamente ciò che si pone volontariamente fuori da quell'ambito. Per ora con questo intervento Dipper ci ha solo detto quello che personalmente pensa della storiografia contemporaneistica italiana. La cosa è molto istruttiva e forse anche emblematica. Manca però la dimostrazione. Secondo le procedure standard quindi il suo contributo non passerebbe la valutazione dei referee. In un'epoca in cui anche l'orientalismo ha già fatto il suo tempo, non bastano più gli avverbi, in qualunque lingua siano scritti, a rendere credibili i luoghi comuni.

\section{Esercizi critici}

\section{Vinzia Fiorino*}

L'occasione è proprio delle migliori: in qualità di componente "straniero" della commissione giudicatrice nella prima tornata di Abilitazione scientifica nazionale (Asn) per il settore di Storia contemporanea, in ossequio alla legge Gel-

* Università di Pisa; vinzia.fiorino@unipi.it 
mini 240/2010, Christof Dipper ha avuto l'opportunità di vagliare ampiamente la più recente produzione storiografica del nostro paese. Profondo conoscitore $\mathrm{e}$ lettore attento della storiografia italiana, ne scrive in un articolo che non tralascia di prendere in considerazione neppure la produzione dei professori ordinari, ovviamente fuori dalle procedure e dagli obblighi concorsuali.

Le argomentazioni si snodano attraverso due principali direttrici fortemente intrecciate: la prima riguarda la politica della ricerca, la sua organizzazione, le modalità di supporto; la seconda passa in rassegna gli aspetti più strettamente qualitativi della produzione scientifica. Cercherò di prendere in considerazione, in forma interlocutoria, alcuni tra i tantissimi temi sollevati.

Non è mai inutile ricordare, se pur molto nota, l'annosa questione dello scarso investimento che i vari governi italiani da sempre hanno riservato alla ricerca scientifica; le università italiane dispongono strutturalmente di risorse economiche relativamente contenute se comparate a quelle degli altri paesi europei.

A questo aggiungerei, entrando subito nel merito degli aspetti più concreti della questione, che spesso riusciamo persino a spendere male le poche risorse disponibili. Una burocrazia sempre più inutilmente farraginosa - la cui farraginosità è esponenzialmente cresciuta in questi ultimi anni —, un personale amministrativo forse non sempre pronto alla ricezione dei nuovi standard, l'imposizione di tempistiche che sembrano del tutto ignorare le esigenze della ricerca e dello scambio internazionale, impediscono il pieno raggiungimento di obiettivi più compiuti e del tutto alla nostra portata. È solo il primo esempio di un'esigenza che vorrei esprimere: la possibilità di una seria programmazione del lavoro scientifico e della realizzazione di veri progetti di ricerca collettivi. Sensibilmente ridotti i Progetti di ricerca di interesse nazionale (Prin), per i settori umanistici non sono rimaste che poche altre possibilità come, in alcuni casi, i più modesti finanziamenti di ateneo. Un contesto così frammentato non può che sollecitare progetti di scarso respiro, spesso improvvisati e confezionati ad hoc in vista, per l'appunto, di un fugace finanziamento. Sfuggire al provincialismo è pertanto quasi impossibile.

Altrettanto nota, e altrettanto profonda, è la ferita inferta all'accademia italiana dalle sue stesse pessime strutture: la didattica avviene in luoghi e contesti spesso non idonei, annosi restano i problemi edilizi, le biblioteche sono spesso poco fornite, e così via. Un'immagine a tutti ben nota: le aule stracolme di alcuni dipartimenti della Sapienza di Roma assunte da tempo a paradigma delle endemiche carenze strutturali dell'accademia italiana, che a ragione Dipper non manca di ricordare. A ciò lo studioso tedesco aggiunge una critica molto serrata a una qualità della didattica del tutto scadente non solo in conseguenza delle strutture poco adeguate, ma anche della scarsa dedizione dei docenti che "recitano dai loro libri di testo". Al riguardo, opterei — però — per una lettura meno uniforme e definitiva, poiché ritengo che la realtà sia molto più differenziata e "a macchie di leopardo": non tutte le strutture (aule, biblioteche, laborato- 
ri ecc.) sono in condizioni disastrose, così come non mi sentirei di condividere la generica e tranchant affermazione secondo cui la didattica in Italia abbia una qualità molto scarsa. La realtà è per l'appunto molto diversificata e non segue affatto una linea che divide nettamente il Nord dal Sud; le aree meridionali possono vantare - nonostante non raggiungano mediamente livelli elevati nelle valutazioni correnti e scontino annosi problemi sistemici - singoli docenti di straordinaria capacità, fortemente integrati nei più avanzati e sofisticati dibattiti storiografici internazionali. Il problema è che spesso si tratta di singoli docenti o di piccoli gruppi di docenti che agiscono in un contesto penalizzato e al quale non si è riusciti ad offrire risposte sistemiche. A conferma di tutto ciò è sempre bene ricordare che se i laureati italiani riescono ad essere competitivi e vengono riconosciuti e apprezzati all'estero, come l'autore non manca di ricordare, è evidente che il sistema formativo italiano è ad oggi in grado di assicurare certi livelli di qualità. Direi anzi, sempre volgendo lo sguardo ai più giovani che si affermano all'estero, che talvolta continua a funzionare una buona integrazione tra ottimi licei e buoni dipartimenti universitari dove - anche su questo punto sfumerei le critiche di Dipper - è del tutto attivo il circolo virtuoso tra ricerca scientifica svolta e programmi didattici proposti.

Per tornare alle questioni strutturali, vorrei cogliere l'occasione, invece, per aggiungere, con rammarico, la grave arretratezza dell'edilizia scolastica e universitaria a far proprie talune scelte di natura ambientalista: nonostante negli anni non sia stata così irrilevante in Italia il dibattito e la sensibilità green, le ristrutturazioni edilizie sono ancora incentrate su ampi e anacronistici sprechi: pochi, se non del tutto inesistenti, i pannelli solari, nessun uso oculato del riscaldamento e dei nuovi sistemi di illuminazione; conseguentemente alte, nei bilanci, le relative voci di spesa. Qui si dispiega un paradosso: è ovvio che nelle università vengano studiati e messi a punto le più sofisticate tecniche e i migliori strumenti capaci di ridurre l'impatto ambientale e contenere i costi di gestione delle strutture, ma la realizzazione nelle stesse strutture è davvero inadeguata. La stessa comparazione con la Germania su questo punto è per l'Italia umiliante. Sarebbe un nobile obiettivo se le università italiane divenissero sotto questo profilo un esempio per il Paese, se i relativi risparmi venissero investiti nella ricerca, se il corpo docente avesse la sensibilità di porre questi aspetti nelle riflessioni pubbliche; mi duole constatare, al contrario, un grande e lungo silenzio al riguardo.

Il contributo di Dipper ripercorre le principali riforme degli scorsi decenni che poco e male hanno inciso sulla qualità del sistema italiano: nel complesso importante la riforma Ruberti, ma, come era stato previsto, del tutto incapace di far giungere ai settori umanistici significativi contributi da parte di gruppi privati. E soprattutto, problema principale, il nostro sistema appare incapace di rinnovarsi, di immettere nuovi e più giovani docenti e ricercatori. Questo è un altro punto cruciale su cui la critica di Dipper è giustamente impietosa. Al riguardo direi che abbiamo scontato e stiamo finendo di scontare una scelta scel- 
lerata compiuta nel 1980 (Dpr 382/1980) e passata nel gergo accademico come "ope legis" (espressione tecnicamente non esatta, ma accettabile nella sostanza) che ha visto, come è noto, l'ingresso in massa di un certo numero di precari del tempo senza una reale selezione. Il provvedimento, ripeto a mio giudizio scellerato, perché umiliante per coloro - tanti — che legittimamente avrebbero potuto vincere una qualsiasi selezione comparativa e disastrosamente generosa per altri che già al tempo avevano dimostrato ridotte inclinazioni per la ricerca, ha avuto un esito perverso: ricoperti ampiamenti tutti i posti possibili, per decenni ha quasi del tutto bloccato il normale e fisiologico turn over. Da lì a poco la generazione successiva di studiosi, fortemente penalizzata, ha cominciato a varcare le frontiere nazionali; pratica che nel tempo è stata esponenzialmente intensificata come effetto di un sistema di reclutamento di fatto bloccato e di risorse costantemente limitate. La più recente riforma Gelmini nella sua prima applicazione, combinata con le sollecitazioni dei vari atenei volte al contenimento della spesa, ha permesso in buona parte una progressione di carriera interna, ma non ha affatto favorito, ancora una volta, nuovi ingressi. Il numero di giovani studiosi che si trasferisce all'estero non può pertanto che continuare a crescere. Inutile sottolineare che il flusso è purtroppo solo "in uscita": il fenomeno non è infatti compensato da un processo inverso, ossia da un reciproco scambio di giovani ricercatori. Le capacità attrattive dell'università italiana sono davvero modeste: pochissimi i docenti che giungono in qualità di visiting e che svolgono cicli di lezioni o di seminari, così come limitate mi sembrano (sempre con le dovute eccezioni) le parternship tra i nostri centri di ricerca interdipartimentali o interuniversitari e quelli stranieri. Indicherei questo aspetto, così cruciale per una vera internazionalizzazione della ricerca e della didattica nonché per una reale sprovincializzazione dell'università italiana, come obiettivo prioritario per una inversione di rotta. Qualche strumento giuridico in più rispetto al passato è ora disponibile ma, come sempre, non riusciamo a metterlo bene a frutto.

Altro capitolo fondamentale delle difficoltà che vive l'università italiana, e su cui mi sembra di notare un deplorevole peggioramento, riguarda la nuova organizzazione del dottorato: istituite le scuole di dottorato in pressocché tutti i dipartimenti sulla base di un accorpamento di discipline affini, si dispiega un grave paradosso: i dottorandi in molte sedi devono frequentare un certo numero di lezioni frontali, ma una quota consistente di esse è di disciplina diversa rispetto a quella propria dei dottorandi. Mi chiedo: ha senso imporre a un livello così alto di formazione, quale è quello di dottorato, un certo numero di ore di didattica dedicate a discipline altre da quelle su cui ci si accinge a scrivere una tesi di dottorato? Anche in questo caso la qualità delle scuole è alquanto differenziata: se per un verso la pratica della cotutela con istituzioni straniere costituisce indubbiamente una nota di merito, dall'altro ho potuto spesso constatare che taluni dottorandi vengono seguiti da tutor con competenze molto diverse dall'argomento della tesi, con evidenti conseguenze negative sul risultato fina- 
le. Anche in questa direzione, avremmo bisogno di una organizzazione didattica più coerente e razionale, una maggiore flessibilità nella scelta dei tutor, una maggiore qualificazione del titolo. Al di là delle prospettive di carriera o della scelta/ripiego di percorrere altre vie professionali, la tesi di dottorato, fuori da ogni retorica, rappresenta un'esperienza fondamentale nell'alta formazione e pertanto credo non si possa prescindere da taluni requisiti: ampia ricerca sulle fonti, capacità di produrre una discussione critica sulla più aggiornata bibliografia, una chiara scelta di tipo metodologico, quindi un apporto significativo alla ricerca sul tema prescelto. Con molte eccezioni, spesso legate alle capacità individuali o alla qualità di alcune scuole particolarmente ben strutturate, direi che siamo abbastanza lontani da questi obiettivi.

Le considerazioni sul dottorato, mi immettono agevolmente al centro degli aspetti qualitativi della produzione storiografica italiana su cui Christof Dipper avanza critiche molto puntuali. Condivido totalmente la prima di esse: più che in passato - afferma Dipper - gli storici esprimono la "necessità di fornire motivazioni teoriche per la scelta dei temi e per l'impalcatura della ricerca" (p. 16). L'Italia gli appare, a buon diritto, del tutto estranea a questo trend. In effetti negli ultimi decenni è quasi del tutto scomparsa nel nostro Paese l'attenzione alle "questioni di metodo"; centrale nel passato, la materia è ora spesso addirittura respinta e rubricata come inutile questione teorica (leggi: filosofica, sofisticata, astrusa) e per di più esclusa dai corsi di studio. I nuovi riassetti e le nuove definizioni dei corsi, avendo cancellato molti insegnamenti a favore di una concentrazione nei corsi fondamentali (storia medioevale, moderna e contemporanea), hanno di fatto eliminato questa tipologia di insegnamenti, in passato spesso crocianamente denominati Teoria e storia della storiografia. Rischiamo dunque di laureare in storia studenti che non hanno mai sentito il nome di Marc Bloch o di Lucien Febvre.

Personalmente ho sempre maturato uno specifico interesse per le questioni di metodo, che continuo a ritenere fondamentali e imprenscindibili sia nel lavoro di ricerca, sia nella comprensione di un testo storiografico. In entrambi i casi reputo infatti ineludibile le domande attorno alla scelta delle fonti, agli interrogativi posti alla ricerca, alle opzioni metodologiche prescelte. La trattazione dell'argomento richiederebbe un intervento ad hoc, tuttavia mi preme sottolineare che la scomparsa di questo interesse è la spia di una precisa involuzione che il nostro Paese ha conosciuto negli ultimissimi decenni: crollati non pochi paradigmi interpretativi, fra cui quello marxista, che è stato particolarmente seguìto in Italia e ha segnato un'ampia stagione storiografica, ci si è talvolta abbandonati ad un bisogno di ricerca archivistica, quasi che le fonti, spesso ingenuamente poste come autentiche depositarie di verità, supplissero e correggessero approcci del passato percepiti come ideologici, quando non non addirittura furvianti. Eppure un certo afflato positivista continua a nutrire molta storiografia italiana: significativa è al riguardo l'ostracismo posto alla storia culturale che infatti in Italia, con le debite eccezioni, non è particolarmente 
praticata. Su uno sfondo molto lontano vi è l'eco di un importante capitolo filosofico che ha visto la contrapposizione tra realisti e costruttivisti, in una prospettiva più ravvicinata vi sono molti storici che continuano a identificare il lavoro storiografico essenzialmente come ricostruzione di fatti e di processi reali contrapposta ad un approccio che, in estrema sintesi, vede nella costruzione discorsiva, visuale, culturale per l'appunto, la fonte privilegiata per cogliere i processi storici reali come sintesi di un un gioco più complesso tra lettura/costruzione dei fatti, delle categorie, dei concetti da un lato e cambiamenti prodotti dall'altro. L'accusa di confondere i fatti con la loro rappresentazione, come se i due aspetti fossero chirurgicamente separabili e distinti a fortiori, di limitare lo sguardo alle classi colte dunque alte (su questo aspetto è evidente un deficit filologico dal momento che la storia culturale nasce con gli studi sull'impatto dei media degli anni Sessanta sulle classi lavoratrici), di produrre una storiografia poco fondata sulle fonti archivistiche e dunque di sminuire la valenza collettiva in passato attribuita alla "Storia", le critiche più frequenti e anche più retrive. Posta la legittimità di ogni opzione metodologica e l'opportunità di mantenere vivace il dibattito, resta difficile non accettare la critica che comunemente ci viene mossa dai colleghi stranieri circa una certa stereotipìa della riflessione italiana, che continua ad essere ingessata e irrigidita sui binari più consolidati della storia politica, che peraltro raramente riesce a spingersi oltre gli anni Settanta. Per suffragare questa critica, Dipper, che pur riconosce quanto il "piacere della provocazione" non sia neppure una regola della storiografia tedesca, cita la scelta finalmente non convenzionale della Società degli storici che in Germania ha premiato una tesi di dottorato sulla prostituzione maschile, chiosando che "di libri del genere [...] non vi è traccia" tra quelli presentati per la selezione concorsuale dell'Abilitazione scientifica nazionale in Italia. Al di là dell'occasione specifica, i temi legati al gender, come dirò più avanti e come lo stesso Dipper riconosce pur non addentrandosi in alcuna riflessione specifica, hanno invece rappresentato, almeno a partire dagli anni Ottanta, un solido filone della ricerca italiana. Citerei piuttosto altri esempi: la storiografia italiana, che per varie ragioni mantiene robuste incrostazioni, presenta limiti ben maggiori legati alle difficoltà di adottare una prospettiva di world history o scarsa propensione a frequentare ambiti di ricerca di confine, meno battuti e che richiederebbero una costante e attiva collaborazione con altre discipline; penso per esempio alla ristrettezza dei visual studies, o ancora l'irresistibile ritrosia a far propri ambiti quali la storia delle emozioni, su cui temo pesi il tipo di ostilità che ho già segnalato per la storia culturale. Tutti ambiti di studio che all'estero e soprattutto in area anglosassone godono di affermati centri di ricerca con specifiche collane, intense attività seminariali di tipo internazionale e ampi scambi (si veda per esempio il Centre for the History of the Emotions con una sede principale presso la Queen Mary di Londra ma con una serie di stabili partnership a Berlino, Sidney e così via). 
Naturalmente non sono sfuggiti a Dipper alcuni filoni storiografici in cui negli ultimi decenni la ricerca è stata particolarmente vivace: come ho già anticipato, la storia delle donne/storia di genere è uno di questi. Indubbiamente si tratta di un filone alquanto ricco e ben riconosciuto all'estero, che negli anni è stato capace di rimettere in discussione periodizzazioni tradizionali, evidenziando la limitata rilevanza - secondo una prospettiva di genere - della tradizionale scansione tra età medioevale/moderna/contemporanea, così come ha cercato di scalzare abbastanza precocemente l'identificazione tra donne e soggetti dominati/subalterni, abbracciando una prospettiva molto più complessa e flessibile che partiva da un ricco confronto critico con le proposte metodologiche di Michel Foucault e di Edward P. Thompson.

Pur non esprimendo particolari sensibilità teoriche, la storia delle donne in Italia, attraverso le indagini che hanno spaziato dalla storia del diritto e della cittadinanza alla storia sociale (ricordo solo, per richiamare qualche esempio, gli studi sulla storia della maternità, su quella del corpo o ancora su quella delle professioni), ha sollecitato un ripensamento critico di categorie solidissime, quali quelle di lavoro, politica, diritti, spazio pubblico. L'adozione della categoria analitica di gender ha quindi avuto implicazioni precise sia nella direzione di un rinnovamento della didattica (significativo l'impegno in questa direzione anche nelle scuole secondarie superiori), sia nella contaminazione con discipline differenti: lo specifico punto di vista dal quale si sono osservati i processi storici ha richiesto infatti un'apertura verso ambiti diversi che spesso hanno investito la storia del sapere scientifico, delle pratiche mediche, la categoria di biopolitica, la storia culturale.

Considerata bizzarra e irrilevante da una storiografia più tradizionalista, ritenuta estranea all'albero genealogico dell'ortodossia da quella più sensibile alla categoria analitica di "classe", la storia delle donne ha a lungo occupato un'area "a parte", così specifica e così circoscritta da non riuscire a raggiungere pienamente uno degli scopi principali che pure si era prefissa: guardare la storia da una prospettiva parziale per poi ripensarla nelle sue conclusioni generali. Resta quindi del tutto attuale, a mio parere, l'auspicio che più storici possano confrontarsi con la categoria di gender e che più storiche possano indagare sui canoni della mascolinità. Non è certo rigorosamente consequenziale, ma neppure del tutto estraneo a questa scarsa circolazione e condivisione di approcci, e non è neppure estraneo a una piena legittimazione dei gender studies, il fatto che, anche nel settore di Storia contemporanea, la presenza delle storiche strutturate sia così fortemente minoritaria. Ad oggi risultano infatti 38 ricercatrici a fronte di 65 ricercatori, 51 professoresse associate contro 93 colleghi associati; la distanza è poi massima nella prima fascia dove la comparazione registra 28 professoresse rispetto a 76 professori ordinari (dati del sito Cineca consultato il 7 gennaio 2017).

Vorrei però avanzare un'ultima considerazione, anche perché mi permette di arrivare ad un paio di riflesssioni conclusive: Christofer Dipper giudica negati- 
vamente la spiccata attitudine al localismo della storiografia italiana. Sì: certamente tante, anzi troppe sono le pubblicazioni di taglio localistico. Gli esempi riportati sono persino grotteschi e, sicuramente, non si può che deprecare l'attività di un/a storico/a incentrata prevalentemente, quando non esclusivamente, su scala locale. Mi chiedo, tuttavia, se sia poi così deplorevole restituire a una comunità la sua stessa storia o la storia di una sua istituzione di rilievo che l'abbia profondamente segnata. E ancora: alcune di queste ricerche realizzate su richiesta e finanziamento degli enti locali (dinamiche peraltro ormai quasi del tutto in via di estinzione) hanno rappresentato il proprio oggetto come un case-study all'interno di un processo storico più ampio, quando non hanno addirittura fatto propri un approccio microstorico, che è stato ovviamente ben altro e molto più che solo una storia locale, come è noto. In taluni casi, inoltre, gli storici alle prese con ricerche condotte su scala locale sono stati sollecitati a partecipare a momenti diversi di confronto pubblico con la comunità indagata. Si tratta mediamente di esperienze di un certo interesse e mi chiedo se non costituiscano anch'esse occasioni per attivare un dialogo e una crescita culturale esterni all'accademia, in grado di non far cancellare totalmente il ruolo culturale e civile dello storico, in grado di evitare l'implosione di una riflessione storiografica che abbia come raggio di espansione soltanto le aule universitarie. In fondo, le stesse considerazioni di Dipper, che in alcuni passaggi sembrano non tenere in debito conto proiezioni e articolazioni che pure la storiografia italiana nel tempo ha saputo stabilire, non vanno in una direzione diversa laddove egli lamenta la mancanza di "un qualsiasi sguardo storiografico a sostegno di un'analisi del tempo presente e, conseguentemente, dei tentativi di risanamento della sfera politica". Obiettivi così alti — che mi sento peraltro di condividere - presuppongono però la riproposizione di molti interrogativi di ampio respiro che non riguardano certo solo l'Italia. È una sfida importante pensare di offrire uno spessore storico ad "un'analisi del tempo presente": una sfida, o un esercizio, che significa contrastare una tendenza ormai da tempo impostasi che presenta la realtà come piatti fotogrammi privi di temporalità; un esercizio che implica per gli storici e per gli intellettuali tout court trovare un proprio ruolo e una propria cifra nel dibattito pubblico che non sempre hanno guadagnato; una sfida, o un esercizio, che prevede l'abbandono di un habitus che ha incasellato l'intellettuale in una dinamica di comunicazione tutta performativa e lo ha ripiegato in un narcisismo solipsistico.

Temi importanti, questi, ma non solo italiani. 


\section{Dal crinale del pre-ruolo}

\section{Antonio Bonatesta*, Andrea Claudi**}

\section{La modernizzazione funzionale del sistema accademico italiano ${ }^{1}$}

Il nostro punto di partenza in questo confronto con il contributo di Christof Dipper è una riflessione sull'atteggiamento delle classi dirigenti italiane nei confronti del sistema dell'istruzione e della ricerca.

Una larga parte della critica ha posto l'accento sulla scarsità di risorse e questo costituisce, indubbiamente, una parte rilevante della questione ${ }^{2}$. È innegabile che la polemica sulle assegnazioni statali alle università esprima in termini più espliciti di altri il conflitto politico e culturale esistente in Italia circa i provvedimenti per la ricerca e il livello di autonomia da assicurare al sistema accademico. Solo alcuni osservatori, tuttavia, hanno adeguatamente problematizzato il nesso di funzionalità che tiene assieme il cosiddetto "sotto-finanziamento" dell'università italiana e le riforme di governance che l'hanno riguardata negli ultimi dieci anni. Non si tratta infatti solo del fatto che "il denaro è utilizzato per altre cose". La sensazione è che la condizione finanziaria di larga parte degli atenei non possa essere spiegata solo ricorrendo alle diverse priorità dei governi o all'esigenza di conservare l'integrità del bilancio statale dopo l'esplosione della crisi del 2008. In poche parole, l'esiguità di risorse ha costituito il prerequisito necessario di un progetto di "modernizzazione dall'alto" dell'università italiana.

A partire dai tagli lineari al bilancio statale della legge 133/2008 e, con maggior forza, dopo la legge 240/2010, l'attenzione dei critici è andata di volta in volta alla penetrazione degli interessi privati nelle università; al loro ruolo nel compromettere l'equilibrio tra scienze "dure" e saperi umanistici, tra ricerca applicata e ricerca di base; alla volontà di colpire uno dei settori della società ancora in grado di esprimere forme di pensiero critico e al declino economico italiano quale scenario di fondo del "suicidio" delle università. Si tratta di spiegazioni che non sempre hanno tenuto conto dell'aspetto che ci pare centrale: i vincoli prodotti dal contesto economico internazionale e dalle forme della competizione globale. Vale a dire, il modo di stare del nostro sistema produttivo e finanziario nell'economia mondiale e il contributo che istruzione e ricer-

* Università del Salento; segretario@dottorato.it

** Università politecnica delle Marche; andrea.claudi@dottorato.it

${ }^{1}$ Nell'ambito di un testo condiviso Antonio Bonatesta è autore dei primi tre paragrafi e Andrea Claudi del quarto paragrafo.

${ }^{2} \mathrm{Si}$ veda tra gli altri Fondazione Res, Università in declino. Un'indagine sugli atenei da Nord a Sud, a cura di Gianfranco Viesti, Roma, Donzelli, 2016; Redazione Roars, Università 3.0: quattro anni vissuti pericolosamente, Roma, Manifestolibri, 2015. 
ca sarebbero chiamate a dare. È solo guardando da tale prospettiva che si può contemplare appieno la continuità con cui sono state sviluppate le politiche per la ricerca da almeno quattro governi a questa parte senza cadere nella trappola narrativa del complotto delle classi dirigenti e dei "poteri forti" alle spalle dell'accademia - leitmotiv di un segmento consistente dei critici "dall'interno" - e senza necessariamente derubricare la legislazione universitaria a mero "gioco della politica".

A nostro parere, i provvedimenti sulla ricerca italiana hanno attraversato in questi ultimi decisivi anni tre fasi sostanziali. Una prima fase, dal 2008 al 2010, ha assistito al consolidamento di un modello egemone nel governo dell'università, basato sulla rottura dei meccanismi solidaristici garantiti dalla spesa pubblica centrale e sull'introduzione di logiche competitive all'interno del sistema accademico. In una seconda fase, nel quinquennio 2010-15, il modello egemone si è costituito in sistema di governance, liberando un processo di "accumulazione primitiva" a favore di alcuni poli e a scapito di altre realtà accademiche regionali (segnatamente quelle del Mezzogiorno). Il "terzo tempo", la fase attuale, è infine quello della piena funzionalizzazione di un sistema ormai polarizzato alle finalità della competizione economica internazionale.

La prima osservazione che ci viene di formulare attorno allo scritto di Dipper, apparentemente più semplice e scontata, è che gli studi storici e segnatamente quelli contemporaneistici stanno dentro queste tre fasi e che dalle loro dinamiche dipendano molte cose. In primo luogo, ruolo e prospettive future della disciplina nel panorama scientifico e culturale del Paese. In secondo luogo, il rapporto che essa è in grado di instaurare con i territori, con il mercato e con lo Stato. Terzo, la possibilità stessa di reiterare canoni, criteri e metodologie proprie della storiografia - non solo di quella italiana - nel momento in cui essa è chiamata a definirsi non più in relazione alle alterne vicende dello Stato-nazione ma in rapporto al mercato. Infine, occorre tenere in considerazione le spinte centrifughe introdotte nella professionalizzazione della disciplina dalla riorganizzazione del "potere accademico" in Italia, che produce come risultato schiere di non-strutturati chiamati a condurre forme e pratiche della ricerca storica su basi completamente diverse da quelle dei decenni trascorsi.

L'assunto è che occorra partire da questa serie di considerazioni per completare una descrizione della storiografia italiana — quale traspare dal giudizio offerto da Dipper - come affetta da "autoreferenzialità", "provincialismo" e "tradizionalismo metodologico". Lungi dal voler difendere gli aspetti deteriori di tali manifestazioni, vale la pena chiedersi se esse siano unicamente addebitabili ai caratteri intrinseci della mentalità italiana/mediterranea e alla strutturazione dell'università di massa in Italia, o se anche i provvedimenti su università e ricerca di questi ultimi anni non stiano piuttosto contribuendo a esasperarne $i$ motivi, anche quando dovrebbero invece eliminarne gli effetti. 


\section{Le conseguenze della polarizzazione del sistema accademico sulle nuove generazioni di storici}

Come detto, dal 2008 il sistema universitario italiano è stato interessato da molteplici interventi sul piano della gestione finanziaria, dell'organizzazione dell'offerta formativa e del reclutamento. L'ultimo in ordine cronologico risale alla legge 240/2010, preceduto e poi accompagnato dalla riduzione di oltre il $15 \%$ del Fondo di funzionamento ordinario (Ffo) e da una continuativa limitazione del turn over ${ }^{3}$. La spiegazione della debole reazione del sistema universitario italiano a otto anni di forte compressione è da ricercare in seno alla classe docente. Come è stato sottolineato, all'interno della comunità accademica italiana è in atto un conflitto "che non è semplicemente fra professori e governo ma fra professori e altri professori sull'idea di università"4.

Sotto la spinta della crisi, provvedimenti come la riorganizzazione del reclutamento, la Valutazione della qualità della ricerca (Vqr) e le nuove forme di distribuzione dei finanziamenti a essa collegate, hanno costituito gli strumenti attraverso cui una parte della classe docente ha governato il processo di ricollocamento dell'università italiana da un contesto di risorse stabili a uno di risorse scarse, assoggettandovi il resto della comunità accademica. Una volta fissata la variabile indipendente, e cioè un regime di bassi finanziamenti, la posta in gioco non è stata la sostenibilità complessiva del sistema ma la trasformazione radicale della sua distribuzione territoriale attraverso una competizione interna per la definizione dei poli egemoni ${ }^{5}$. A tale esito non sembra essere stato del tutto estraneo il confronto sviluppatosi in seno alla Conferenza dei rettori (Crui), organismo che tra il 2008 e il 2010 ha conosciuto una forte contrapposizione tra due "correnti" associative interne espresse dall'Associazione per la qualità delle università italiane statali (Aquis) e dalla Rete degli atenei meridionali (Ram) ${ }^{6}$. Entrambi i raggruppamenti di atenei hanno avuto vita breve ma ciò che qui rileva è che, in quel frangente, essi erano portatori di istanze e interessi territorialmente contrapposti: Acquis aggregava gran parte degli atenei settentrionali e i politecnici con lo scopo di "contribuire a innalzare la competitività internazionale dell'università italiana"7; Ram consociava gli atenei me-

${ }^{3}$ Negli ultimi otto anni i finanziamenti pubblici all'università sono scesi da 7.443.700.000 a 6.904.800.000 di euro, mentre il personale di ruolo ha subìto una contrazione di oltre 7.800 unità tra docenti ordinari, associati e ricercatori, Fondazione Res, cit.

${ }^{4} \mathrm{Si}$ veda Roberto Ciccarelli, Università, Stefano Semplici: "La valutazione è a rischio, cresce la protesta», "il manifesto", $1^{\circ}$ marzo 2016,

${ }^{5} \mathrm{Si}$ veda Antonio Bonatesta, Il reclutamento impossibile. Il precariato nell'Università italiana raccontato senza "casi esemplari", "Analysis. Rivista di cultura e politica scientifica", 2015 n. 1, pp. 1-7.

${ }^{6} \mathrm{Si}$ veda Carmelo Pasimeni, Prove di riforma e ruolo delle Università meridionali nel divario nord-Sud del paese (2008-2010), "Itinerari di Ricerca Storica”, a. XXX, 2016 n. 1, pp. 161-182.

${ }^{7}$ Pasimeni sostiene che "il vero obiettivo dell'associazione, però, [sia stata] la messa in discussione del modello corrente di ripartizione del fondo ordinario che, in un contesto di tagli al- 
ridionali e dal 2010 faceva affidamento sul Forum delle Università del Mezzogiorno costituito in seno alla Svimez ${ }^{8}$.

L'esito di quel confronto è sfociato in un nuovo modello di gestione dell'università italiana. La creazione della nuova struttura del "potere accademico", basata sulla divisione tra nuclei "forti" e nuclei "deboli", è passata attraverso la programmazione della divergenza del Mezzogiorno - e di altre aree periferiche del Centro e del Nord-Est - rispetto a un reticolo policentrico egemone collocato prevalentemente nelle maggiori regioni settentrionali. Se nel secondo Novecento la convergenza tra le diverse aree territoriali era in Italia un obiettivo dell'intervento pubblico e della programmazione democratica, oggi la divergenza ha assunto le sembianze di un processo di "accumulazione primitiva" che necessita di strumenti tecnocratici e di retoriche meritocratiche utili alla propria legittimazione. Le modalità di distribuzione dei punti organico - alla base della definizione delle possibilità assunzionali dei singoli atenei —, la formazione di alcuni indicatori della Vqr e l'adozione di parametri come il cosiddetto "costo standard" nell'allocazione della quota base del Ffo sono strumenti ascrivibili alla tecnica della "divergenza programmata", praticata principalmente a detrimento del sistema accademico meridionale .

In base alle disposizioni di legge, l'Ffo è ripartito tra una "quota premiale" distribuita tramite la Vqr - che nei prossimi anni sarà condotta al suo limite massimo del 30 per cento del totale - e una "quota base". Una prima considerazione da fare è che ogni "premio" per un ateneo si traduce in una sottrazione per un altro: questo effetto distorsivo non si avrebbe se la quota premiale fosse davvero aggiuntiva rispetto allo stanziamento ordinario. In secondo luogo, a partire dal 2015 una parte sempre maggiore della quota base è progressivamente allocata tenendo conto non più delle assegnazioni storiche per ogni singolo ateneo ma di un "costo standard" per studente, formato dalla somma di vari addendi standardizzati su base nazionale. Sebbene tra questi sia computato anche un indice perequativo regionale, costruito in base all'esigenza di compensare le università della minor leva contributiva studentesca in regioni dove il reddito medio familiare è più basso, il suo effetto è tuttavia molto contenuto ${ }^{10}$. Così il "costo standard" si combina con i più bassi livelli di ricchezza e con la

la spesa pubblica, non poteva più essere distribuito a tutti gli atenei secondo i vecchi parametri indipendentemente dal livello della qualità e dell'efficienza della gestione", ivi, p. 174.

${ }^{8}$ L'esperienza della Rete delle università del Mezzogiorno non riuscì a svincolarsi dai mandati dei rettori che l'avevano promossa. Come è stato osservato, nonostante l'assertività di alcune proposte essa si è collocata prevalentemente sulla "difensiva", dovendo far fronte alle accuse di "gestione facile" delle università meridionali, ivi, p. 173.

${ }_{9}$ A tal proposito si veda Antonio Bonatesta, Questione meridionale e "divergenza programmata" nell'università italiana: la prospettiva del reclutamento accademico, "Itinerari di Ricerca Storica", a. XXX, 2016, n. 1, pp. 183-190.

${ }^{10} \mathrm{Si}$ veda Manuela Ghizzoni, Finanziamento delle università e costo standard: dal senso comune al buon senso, www.manuelaghizzoni.it, 16 maggio 2016. 
dinamica demografica negativa del Mezzogiorno ${ }^{11}$, oltre che con il calo degli immatricolati nelle università meridionali ${ }^{12}$, e potrebbe presto condurre a un'ulteriore chiusura di molti corsi di laurea a carattere scientifico e specialistico ${ }^{13}$. Chiudere corsi significa perdere studenti, perdere studenti significa dover rinunciare a risorse di bilancio, al reclutamento, significa ridimensionarsi.

A partire dalla legge 240/2010, anche il reclutamento di nuovi ricercatori ha dovuto mutare il suo rapporto di funzionalità con la riproduzione del sistema accademico. Al reclutamento è stato affidato un compito complementare a quello dei pensionamenti: mentre questi smaltiscono la vecchia struttura demografica, l'immissione in ruolo è rigidamente limitata dai blocchi parziali del turn over e dalla ripartizione dei punti organico. Nemmeno questi freni si sono rivelati neutrali rispetto alla distribuzione territoriale del reclutamento. Ne sono derivati fortissimi squilibri: in base ad alcuni calcoli, è come se negli ultimi quattro anni quasi 700 ricercatori siano stati prelevati dagli organici delle università del Centro-Sud e trasferiti negli atenei dell'Italia settentrionale ${ }^{14}$.

Il modo in cui i provvedimenti della "divergenza programmata" hanno inciso sulla formazione dottorale e sul reclutamento di nuovi ricercatori conserva un'importanza fondamentale per comprendere appieno le conseguenze della concentrazione delle risorse in pochi poli.

Il dottorato italiano, sul quale Dipper ha poche ma non del tutto positive parole, è andato incontro a una diminuzione del $44,5 \%$ in dieci anni, passando da 15.733 posti a bando nel 2006 a 8.732 nel 2016. Eppure l'Italia era ed è agli ultimi posti in Europa per numero di dottorandi ogni mille abitanti, in base a un indicatore che la collocava in fondo alle graduatorie europee già nel 2012 e che nel 2014 ha subìto un ulteriore ridimensionamento (da 0,58 a 0,56 dottorandi ogni mille abitanti) ${ }^{15}$. Ben sette regioni italiane garantiscono il 74,5\% dell'offerta dottorale italiana (Lazio 18\%, Lombardia 17,5\%, Campania 9\%, Toscana $8,5 \%$, Emilia Romagna 8,5\%, Veneto 7\%, Piemonte 6\%), un dato che tiene an-

${ }^{11} \mathrm{Si}$ veda Svimez (a cura di) Rapporto 2015 sull'economia del Mezzogiorno, Bologna, il Mulino, 2015.

${ }^{12} \mathrm{Si}$ veda Anvur, Rapporto sullo stato del sistema universitario e della ricerca 2016, (versione sintetica), 24 maggio 2016, http://www.anvur.org/attachments/article/1045/Rapporto_ANVUR_SINTESI_20 .pdf., p. 14 e ss.

${ }^{13}$ In base alle stime dell'Anvur, la contrazione nel numero dei corsi si è finora verificata principalmente in seguito alla riduzione delle sedi decentrate: i corsi sono diminuiti del $17,1 \%$ al Nord, del 22,6\% al Sud e del 28,2\% al Centro, ivi, p. 12.

${ }^{14} \mathrm{Si}$ veda Bruno Cappelletti Montano, Punti organico: in 4 anni il Nord si è preso 700 ricercatori dal Centro-Sud, 18 agosto 2015, http://www.roars.it/online/punti-organico-una-propostache-si-puo-rifiutare/.

${ }^{15} \mathrm{Nel} 2012$ l'Italia al terzultimo posto tra i paesi europei con 0,6 dottorandi ogni 1.000 abitanti. Si veda Alessio Rotisciani, Stefania Napoli, I migliori standard europei? Il confronto con le altre realtà nazionali, Quarta Indagine annuale ADI su dottorato e post-doc, 2014, https://dottorato.it/sites/default/files/survey/V\%20Indagine\%20ADI\%20-\%20I\%20migliori\%20standard\%20 europei $\% 2011 \% 20$ confronto\%20con\%20le\%20altre\%20realta\%20nazionali.pdf. 
che se pesato sulla numerosità delle rispettive comunità accademiche regionali. L'espressione più conforme di questa situazione è che dieci atenei settentrionali garantiscono da soli il $44 \%$ di tutte le posizioni bandite. Parallelamente, per effetto del decreto ministeriale n. 45 dell'8 febbraio 2013, si è registrata una contrazione del numero di corsi di dottorato, passati dagli oltre 1.500 del 2013 ai 900 del $2014^{16}$. È questo uno dei dati che deve maggiormente far riflettere, dal momento che nella scomparsa per soppressione o accorpamento di 600 corsi di dottorato è compreso di tutto: certamente l'eliminazione di ridondanze e di nicchie di "potere accademico" ma, anche e soprattutto, la possibilità di istituire curricula specifici sotto l'aspetto scientifico-disciplinare. Sarà estremamente improbabile che tali effetti possano consentire il dispiegamento delle potenzialità della rierca storico-contemporaneistica, costretta in molti dottorati a convivere con altre discipline all'interno di corsi melting pot.

In questo solco ha agito anche la disarticolazione del percorso di formazione-avviamento alla ricerca-accesso ai ruoli accademici, provocato dalla messa in esaurimento della figura del ricercatore a tempo indeterminato $(\mathrm{Ru})$ e dalla sua sostituzione con quella del ricercatore a tempo determinato di tipo "a" (Rtda) e di tipo "b" (Rtdb). La legge 240/2010, infatti, ha interposto tra il dottorato e l'accesso al primo gradino della struttura accademica - la posizione di professore associato - una moltitudine di figure e contratti precari di durata variabile. Dopo il conseguimento del dottorato, un dottore di ricerca può trovarsi dinanzi alla possibilità di cumulare fino a un massimo di sei anni di assegni di ricerca, prima di avviarsi alla vera e propria trafila del pre-ruolo che consiste in tre anni da Rtda e altri tre da Rtdb.

Questo percorso, qui presentato al netto di periodi di collaborazione alla ricerca e di didattica svolti gratuitamente o in base a contratti avventizi, si fonda sull'istituzionalizzazione di dodici anni di precariato a partire dal conseguimento del titolo di dottorato. La conseguenza più diretta è lo slittamento dell'età media di ingresso nel ruolo degli strutturati, passata dai 36 anni del 2006 agli oltre quarant'anni di oggi, con evidenti conseguenze sull'invecchiamento complessivo del corpo docente ${ }^{17}$. È legittimo avanzare delle perplessità sulla natura di questo intervento. Si è voluto incidere sul livello dei ricercatori a tempo indeterminato, una cui parte considerevole "è costituita — come sottolinea Dipper — da sessantenni senza ulteriori titoli oltre la laurea ma [...] con lo stesso carico di insegnamento dei professori ordinari", per avere in cambio ricercatori a tempo determinato ultraquarantenni spesso overqualified rispetto

\footnotetext{
${ }^{16}$ Alfredo Ferrara (a cura di), Il dottorato di ricerca in Italia: precario stato di salute di un giovane trentenne, Quinta Indagine annuale Adi su dottorato e post-doc, Roma, 2015, https:// dottorato.it/content/v-indagine-adi-su-dottorato-e-post-doc.

${ }^{17} \mathrm{Si}$ veda Anvur, Rapporto sullo stato del sistema universitario e della ricerca 2013, 2014, http://www.anvur.org/attachments/article/644/Rapporto\%20ANVUR\%202013_UNIVERSITA\%20e\%20RICERCA_integrale.pdf.
} 
al proprio status, gravati da carichi didattici e da obblighi di garanzia sui corsi di insegnamento. Risulta pertanto impossibile smentire l'affermazione secondo cui "l'insegnamento basato sulla ricerca è la grande eccezione", sebbene occorra focalizzare adeguatamente le cause del fenomeno.

Precarizzazione e disarticolazione del rapporto tra formazione dottorale e accesso al ruolo rispondono all'esigenza di rallentare la catena di cooptazione delle regioni del sistema destinate a costituire nel prossimo futuro la nuova periferia dell'università italiana, costringendole a orientare le sempre più esigue risorse di bilancio verso forme contrattuali meno costose - borsisti, collaboratori, assegnisti, ricercatori a tempo determinato di tipo "a" - con cui riescono a garantire le attività di didattica e ricerca senza incidere sulla riproduzione dei ruoli accademici ${ }^{18}$.

La distribuzione territoriale delle posizioni da Rtdb - l'unica figura collegata all'ingresso in ruolo - mostrava nel 2014 una spiccata concentrazione nelle regioni centro-settentrionali del Paese. In termini assoluti, ben sette sistemi regionali non hanno bandito alcuna posizione da Rtdb: si tratta di Basilicata, Calabria, Molise, Puglia, Sardegna, Friuli-Venezia Giulia e Valle d'Aosta. Di queste sette regioni, solo le ultime due non sono collocate nel Mezzogiorno. In questo scenario, le prime otto università per reclutamento di nuovi Rtdb detenevano da sole il 51\% dell'intero contingente nazionale di posizioni per il 2014; di queste, solo due appartenevano al Sud ${ }^{19}$.

In questi anni dunque la reazione spontanea della comunità accademica ai vincoli finanziari e normativi è stata di innalzare il tasso di precariato al proprio interno pur di salvaguardare una tenuta, messa a rischio dalla competizione egemonica. In spregio alla promessa di neutralizzare il potere del "baronato" universitario con cui era stata presentata all'opinione pubblica, la riforma del 2010 ha promosso ulteriori concentrazioni di potere e configurato un esercito di riserva di giovani ricercatori, debole perché dipendente e sostituibile. La proliferazione degli organismi di rappresentanza degli interessi sezionali osservata da Dipper - che coinvolge dottorandi, assegnisti di ricerca e ricercatori a tempo determinato passando per una parte dei docenti e del personale tecnico

${ }^{18}$ Come ha rilevato uno studio dell'Associazione dottorandi e dottori di ricerca italiani (Adi), se questi livelli dovessero persistere, dei 14.400 assegnisti attivi nel 2014 il 76,5\% non continuerebbe a fare ricerca dopo aver fruito di uno o più anni di assegno; il 15,4\% uscirebbe dal mondo della ricerca dopo un contratto da Rtda; solo l'8,1\% sarebbe trasformato in Rtdb, avviandosi verso l'ingresso in ruolo. In definitiva, quasi il $92 \%$ sarebbe destinato all'espulsione nel volgere di sei anni. Cfr. Adi - Associazione dottorandi e dottori di ricerca italiani, Quinta Indagine annuale ADI su Dottorato e Post-Doc. Il reclutamento di assegnisti, ricercatori a tempo determinato a e b, 18 giugno 2015, https://dottorato.it/sites/default/files/survey/V\%20Indagine\%20ADI\%20 -\%20I1\%20reclutamento\%20di\%20assegnisti $\% 20$ ricercatori $\% 20$ a $\% 20$ tempo $\% 20$ determinato $\% 20$ di\%20tipo\%20a\%20e\%20b.pdf.

${ }^{19}$ Si tratta del Politecnico di Milano, dell'Università di Messina, dell'Università di Salerno, del Politecnico di Torino, dell'Università degli Studi di Chieti-Pescara, dell'Università di Trento, dell'Università di Camerino, dell'Università di Firenze: ivi. 
amministrativo (quest'ultimo è l'unico in cui sono realmente operative le sigle sindacali) - sarà anche il sintomo della "mentalità assistenziale" di un paese latino ma sembra soprattutto una reazione alle pressioni introdotte nella struttura accademica e allo svuotamento delle istituzioni di rappresentanza come il Consiglio universitario nazionale (Cun). Fin qui, le principali decisioni sono state assunte in base a una triangolazione tra Miur, Anvur e Crui, laddove i primi due sono organismi a carattere burocratico e tecnocratico mentre la Conferenza dei rettori è composta da membri non sempre interessati al mantenimento del consenso nei propri atenei, in considerazione del fatto che a partire dal 2010 il loro mandato non è più rinnovabile.

Le conseguenze dell'insieme di questi processi sull'attività di ricerca sono immaginabili. Non si tratta tanto di svincolare il giovane ricercatore dal controllo dei propri referenti e liberarne la creatività, quanto del fatto che l'elemento sociale e territoriale nel punto di accesso all'esercizio della ricerca è divenuto quanto mai vincolante. Sopportare tanto precariato non è per tutte le tasche e la condizione familiare di provenienza risulta decisiva nel resistere all'instabilità della propria situazione professionale e reddituale. Del resto, il tentativo di dare continuità al proprio percorso influisce sugli stessi indirizzi di ricerca.

Per quanto concerne gli studi storici, e in particolare quelli contemporaneistici, dietro etichette come "provincialismo" e "localismo" o a monte delle denotazioni sulla scarsa chiarezza del confine tra contatti professionali e clientelismo, possono coesistere tanto fenomeni di malcostume quanto il tentativo da parte degli storici non strutturati di reperire risorse sul mercato, tra fondazioni, enti, istituti culturali e case editrici, per garantirsi il prosieguo dell'attività di ricerca. Naturalmente, ogni istituzione accademica si interfaccia con il proprio territorio di riferimento, fatta eccezione per i poli egemonici che assurgono alla realtà di snodi delle reti transnazionali della ricerca, garantendo l'accesso a circuiti più ampi.

Torna così il tema della sperequazione territoriale, se è vero che al localismo della ricerca storica contemporaneistica, specie nel Mezzogiorno, possono contribuire tanto la grande tradizione del meridionalismo quanto la debolezza dell'apparato produttivo e dei circuiti culturali di quest'area del Paese. Tale problema riguarda non solo il Sud se si considerano gli effetti di quella che è stata definita la "regionalizzazione del sistema di finanziamento delle università", in risposta alle spinte polarizzanti della normativa nazionale ${ }^{20}$. Anche in questo caso l'intervento surrogatorio della spesa pubblica da parte di fondazioni bancarie o degli enti regionali finisce col vincolare l'attività di ricerca, reindirizzandola di volta in volta verso il soddisfacimento di interessi particolari o modellandola a ridosso delle destinazioni di spesa dei finanziamenti pubblici o privati.

${ }^{20}$ Carmelo Pasimeni, cit., p. 173.

Copyright (C) FrancoAngeli 
Resta il fatto che, una volta indebolita o compromessa l'azione perequatrice delle risorse statali, agganciare la ricerca al mercato impone condizioni diverse a seconda delle territorialità e, in questo senso, gli studi storici non fanno eccezione. Spesso i giovani studiosi sono costretti a ricalibrare o reinventare i propri interessi di ricerca al fine di partecipare a un bando o a un premio che garantisca loro ulteriori mezzi e opportunità. È però vero che dedicarsi a determinate ricerche con il solo obiettivo di sostenere le condizioni materiali del proprio percorso costituisce una dispendiosa dilapidazione di tempo e di energie. Ne deriva che, nel momento in cui si accetta di "scendere" al rango di semplici operatori e mediatori culturali, al centro della ricerca non vi sia più l'innovazione e la formazione individuale dello storico ma le esigenze del mercato nelle sue varie accezioni. Purtroppo, o naturalmente, i risultati di indagini storiche instradate per questa via difficilmente assurgono al rango di prodotti della ricerca valutabili o apprezzabili in sede di Abilitazione scientifica nazionale, anche a causa dei limitati sbocchi sulle riviste scientifiche - contingentati dalla organizzazione in fasce di qualità da parte dell'Anvur - e della demonizzazione delle storie regionali, locali e di altri specialismi che, dopo essere state assai diffuse, non pare sia stata aliena dai giudizi dei Gruppi di esperti della valutazione (Gev), consolidando nuove "ortodossie" storiografiche. È del tutto legittimo ipotizzare cioè che determinati orientamenti di ricerca tendano a diventare prevalenti all'ombra dei meccanismi di valutazione e possano più facilmente emergere da segmenti tradizionalmente "forti" della storiografia italiana, riflettendo la polarizzazione del "potere accademico".

Il punto è capire se la direzione intrapresa imprima un'azione destrutturante o strutturante di certi caratteri della storiografia italiana, registrati in sede di abilitazione da Dipper.

\section{Il "terzo tempo" delle politiche per la ricerca: emarginazione e autorefe- renzialità degli studi storici?}

Recentemente, alcuni elementi hanno fornito l'impressione che si stia entrando in una fase qualitativamente nuova nella governance dell'università. Le vicende dello Human Technopole di Milano e il provvedimento sulle cosiddette "cattedre Natta" hanno mostrato una tendenza allo sdoppiamento nel sistema di attribuzione delle risorse alla ricerca: una parte è quella tradizionale, stabilizzata su livelli di sottofinanziamento; l'altra è arbitraria, cioè erogata dal governo ad hoc e concertata con gli investimenti privati. Mutano anche gli attori istituzionali: nel "terzo tempo" si sviluppa il protagonismo diretto della Presidenza del consiglio che avrebbe o starebbe commissariando le altre competenze ministeriali.

Ci pare che si possa dire che tali provvedimenti siano dettati dall'esigenza di governare in modo diretto ed efficiente, al riparo da interferenze democra- 
tiche e dalla presunta incoercibilità del sistema accademico verso fini prestabiliti, la risposta che le classi dirigenti italiane stanno costruendo al tema della conflittualità economica internazionale e della competizione industriale globale. Questa risposta, almeno al tempo del governo di Matteo Renzi, aveva un nome: Industria 4.0 (I4.0). Il World economic forum ha previsto che l'impatto delle nuove tecnologie produrrà nei prossimi anni un saldo netto negativo di oltre 5 milioni di posti di lavoro ${ }^{21}$. L'Italia deve rispondere alla sfida di un saldo in pareggio - 200 mila posti creati e altrettanti persi - che tuttavia nasconde perdite nelle aree dei servizi amministrativi e della produzione, compensate da nuovi posti nell'area finanziaria, nel management, nell'informatica e nell'ingegneria. Presentato nel settembre 2016, il piano Industria 4.0 aveva dunque l'obiettivo di riorganizzare il sistema produttivo nazionale attorno al pieno e proficuo utilizzo di macchine intelligenti, interconnesse e collegate a internet, seguendo analoghe iniziative di Stati uniti, Germania e Francia. Tra le direttrici strategiche vi è lo stimolo degli investimenti privati su tecnologie e beni I4.0 e l'aumento della spesa privata in ricerca e sviluppo. Quest'ultimo punto appare decisivo ed esprime il salto di livello rispetto al "secondo tempo": non l'orientamento del sistema accademico verso finalità produttive ma la delega diretta ai super-poli: competence centers e digital innovation hubs.

Qual è il posto dei saperi storici, e in particolare storico-contemporaneistici, in un contesto simile? L'interrogativo risulta ancora più pressante se ci si confronta con le recenti misure che hanno condotto all'introduzione del cosiddetto "dottorato innovativo", destinato ad assorbire una parte cospicua delle risorse destinate alla formazione di terzo livello ${ }^{22}$. Il problema fondamentale - come ha sottolineato recentemente anche il $\mathrm{Cun}^{23}$ - è che in questo caso non si tratta più di fondi aggiuntivi ma di uno spostamento di risorse ordinarie dai dottorati tradizionali a quelli innovativi nell'ordine del $60 \%$ dell'intera quota dell'Ffo $2016^{24}$. Alla luce di questi provvedimenti, non appare del tutto infondata la preoccupazione che dottorati o curricula in Storia o Storia contemporanea possano andare in sofferenza ed essere emarginati, ovvero esclusi dal novero delle discipline strategiche per le prospettive di sviluppo del Paese. Alterando i

${ }^{21} \mathrm{Si}$ veda World economic forum, The Future of Jobs. Employment, Skills and Workforce Strategy for the Fourth Industrial Revolution, 2016, http://www3.weforum.org/docs/WEF_Future_of_Jobs.pdf.

${ }^{22}$ I criteri per la definizione dei dottorati innovativi sono — si ricorda — internazionalizzazione, interdisciplinarità e intersettorialità. In particolare, quest'ultimo criterio riprende la definizione già presente nel documento dell'UE Principles for Innovative Doctoral Training (PIDT) e comporta una stretta connessione con l'industria e con altri rilevanti settori d'occupazione.

${ }^{23} \mathrm{Si}$ veda Consiglio universitario nazionale (Cun), Raccomandazione sulla nota del capo dipartimento del 31.8.2016 avente come oggetto «Dottorati innovativi, attuazione del Programma nazionale per la ricerca 2015-2020 e indicazioni con riferimento al Dm n. 552/2016 (art. 10, comma 1, lett. e)», adunanza del 19 ottobre 2016.

${ }^{24}$ Si veda il Dm 6 luglio 2016, n. 552, "Criteri di ripartizione del Fondo di finanziamento ordinario (Ffo) per l'anno 2016". 
caratteri strutturali del dottorato e spostando ingenti quantitativi di risorse ordinarie verso obiettivi specifici, nel volgere di pochi anni le possibilità di reclutamento di interi settori scientifico-disciplinari potrebbero risultare compromesse.

Tutto ciò accade mentre gli studi storici sono chiamati, da tempo, a elaborare una risposta alla sfida proveniente da altri settori concorrenti - accademici e non - nella costruzione di senso comune storico. Non è sempre diffusa la consapevolezza delle conseguenze derivanti dalla presenza nel discorso pubblico di giornalisti, opinionisti, pubblicitari, operatori culturali e altre svariate figure che promuovono interpretazioni di fatti e processi storici. L'interesse di una parte della comunità degli storici verso i temi della Public History, unitamente all'avvio di percorsi associativi in tale settore, segnala la maturazione del confronto su questo tema. Resta da capire, dinanzi ai condizionamenti prodotti dai fenomeni descritti in queste pagine, il grado di efficacia di risposte basate sulla Public History.

Il problema è profondo e riguarda la crisi di legittimazione del sapere scientifico nella società di oggi e le sue difficoltà a opporsi a revisionismi e negazionismi. Queste difficoltà non sono esclusivamente riconducibili solo a questioni epistemologiche ma riguardano anche le modalità attraverso cui la conoscenza scientifica produce senso comune. Questa specifica forma di produzione è oggi compromessa dalla rottura del rapporto tra intellettuali e politica. In Italia l'intellettualità ha storicamente trovato una propria fonte di legittimazione anche nel rapporto con la politica e questo è valso in particolare per gli storici. Negli ultimi due decenni è stata condotta, sulla scorta della temperie culturale del nostro Paese, una crociata contro la rilevanza delle appartenenze politiche nella storiografia italiana, considerato retaggio della "Repubblica dei partiti". La conseguenza più evidente di tale enfasi, uguale e contraria alle storture che intendeva risolvere, è che allentato o trasformato il rapporto con la politica il sapere e in particolare la storiografia hanno ritenuto di potersi garantire nuove forme di legittimazione attraverso le tecniche di valutazione della qualità della ricerca. È in questo senso, semmai, che il discorso scientifico e con esso gli studi storici rischiano di esprimere una dinamica autoreferenziale. Stretta in questa crisi, la nostra impressione è che la storia stia perdendo le grandi arene di formazione dell'opinione pubblica - televisione e giornali senza aver appreso come occupare le nuove, vale a dire i social media, terreno di confronto decisivo in cui imperversano le architetture di propaganda e all'interno dei quali il pubblico è rinchiuso in "camere di ridondanza" che rafforzano il pensiero radicale $^{25}$. Nel web e nei social media, come anche nella stampa e in tv, il senso storico non si costruisce attraverso i criteri canonici del metodo scientifico né viene validato dai sistemi di valutazione della qualità della ricerca. Pur sen-

\footnotetext{
${ }^{25}$ Walter Quattrociocchi, Antonella Vicini, Misinformation. Guida alla società dell'informazione e della credulità, Milano, FrancoAngeli, 2016.
} 
za negare alla radice il tema della valutazione, la nostra impressione è che oggi mentre i ricercatori sono occupati a scrivere per essere valutati, sui social network il senso storico è sempre più affare d'altri e si produce sulla base di dinamiche di ridondanza e di consenso. Se esiste un problema di autoreferenzialità degli studi storici in Italia, esso potrebbe dunque avere radici ben più profonde di quelle messe in evidenza da Christof Dipper.

\section{Appunti su alcune tendenze strutturali del settore di Storia contempora- nea nella trasformazione dell'università italiana}

Definito il quadro generale della nostra riflessione, è ora possibile accostarsi ad una analisi "dall'interno" del settore M-sto/04 per provare se e quanto le affermazioni di Christoph Dipper "tengano" alla prova dei numeri. Come sottolineato in precedenza, gli effetti dei provvedimenti che — dal 2008 in poi - hanno inciso sulla realtà accademica italiana sono molto profondi. Nei paragrafi che seguono ci limiteremo ad analizzare l'impatto che queste misure hanno avuto su questo settore scientifico-disciplinare ${ }^{26}$.

Consistenza numerica del corpo docente. Nel 2008, i docenti di ruolo nel settore assommano alla cifra di 528. Nel corso degli anni, tale cifra decresce in maniera monotòna, fino ad arrivare a 383 nel 2016, con un calo del $27.5 \%$. Per porre questi numeri nella giusta prospettiva, è utile sottolineare che nel 2008 il totale del personale docente universitario italiano era di 60.594, mentre nel 2016 assomma a 50.635, con un calo del 16.5\% (Fig. 1).

Il settore M-sto/04, dunque, sembra essere stato vittima del processo di compressione selettiva delineato anche da Christoph Dipper, un processo che si esplica non solo sulla dimensione geografica ma anche su quella dei differenti settori scientifico-disciplinari.

Consistenza numerica dei ruoli della docenza. Le misure legislative degli ultimi anni hanno pesantemente modificato il profilo dei ruoli della docenza universitaria. La legge 240/2010 ha infatti posto a esaurimento il ruolo di ricercatore universitario a tempo indeterminato $(\mathrm{Ru})$, per sostituirlo con due differenti contratti di ricerca a tempo determinato.

\footnotetext{
${ }^{26}$ L'analisi ha un tenore marcatamente statistico e quantitativo, e si basa su dati messi liberamente a disposizione dal consorzio Cineca, mediante lo strumento "CercaUniversità": cfr. http:// cercauniversita.cineca.it. L'analisi è limitata agli anni che vanno dal 2008 al 2016, anni nei quali sono dispiegati gli effetti dei provvedimenti sopra citati. Per quanto riguarda gli assegnisti di ricerca, i dati a disposizione non sono purtroppo storicizzati e si limitano a fotografare le posizioni in essere al momento dell'interrogazione del database. I dati, disponibili nel formato proprietario xls, sono stati convertiti nel formato libero csv e successivamente analizzati per mezzo di un software realizzato dagli autori di questo articolo, che ha anche prodotto i grafici utilizzati per questo lavoro. I dati e il software utilizzati per l'analisi, insieme ai grafici del presente articolo sono disponibili al link https://gitlab.com/norbertus/ic-paper. Il software è sottoposto a licenza GPLv3, mentre il materiale grafico è licenziato sotto licenza CC BY-NC-SA 3.0 IT.
} 
Fig. 1

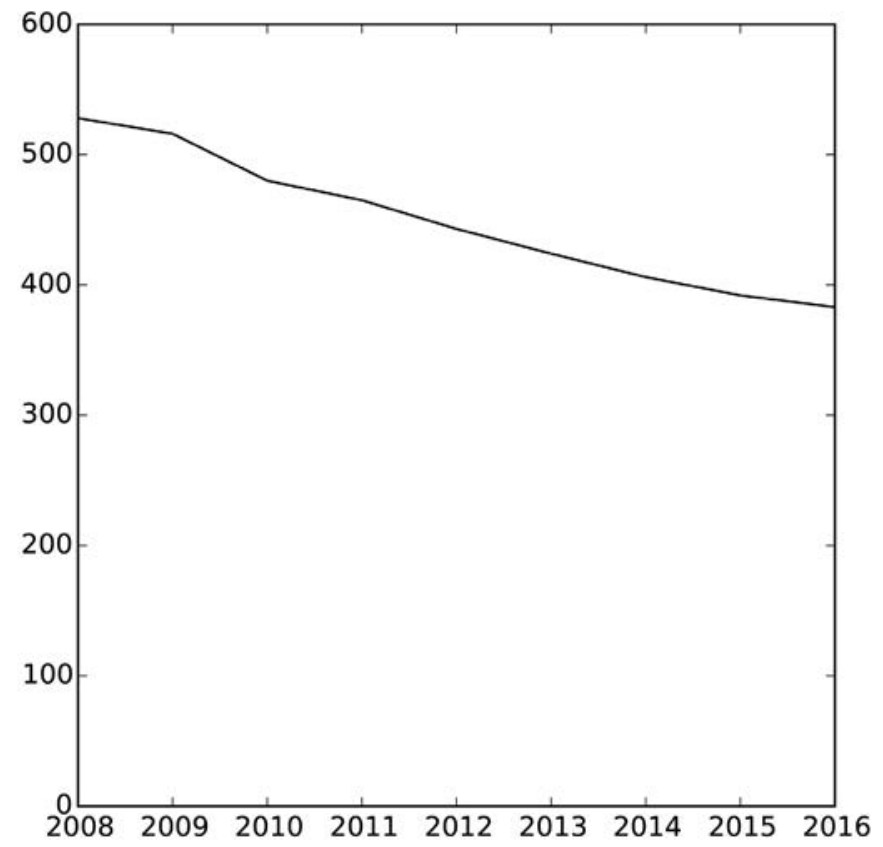

In Fig. 2 il numero totale dei docenti del settore M-sto/04 è diviso nei diversi ruoli: professori ordinari $(\mathrm{Po})$, associati $(\mathrm{Pa})$, ricercatori $(\mathrm{Ru})$ e in ricercatori a tempo determinato (Rtd).

Balza immediatamente all'occhio la flessione nei ruoli di Po e Pa in corrispondenza al 2010, anno di emanazione della legge Gelmini. Per quell'anno i ranghi per i Pa e i Po si riducono rispettivamente di 14 e 17 unità. Sono altresì evidenti gli effetti del Piano nazionale associati, con cui ad un numero rilevante di $\mathrm{Ru}$ viene consentito di accedere al ruolo di $\mathrm{Pa}$; quantificheremo in seguito il numero di promozioni relative a questa misura. Meno evidente, infine, è l'effetto del piano straordinario per l'assunzione di Rtdb del 2016; la consistenza della categoria, molto esigua fino al 2015, giunge quasi al raddoppio solo nell'ultimo anno.

In Fig. 3 la consistenza dei ruoli è analizzata in percentuale sul totale dei docenti, rendendo più evidenti le dinamiche appena evidenziate.

È opportuno notare che la consistenza degli Rtd (compresi gli Rtda, il cui accesso in ruolo non è garantito) è di poco superiore all' $8 \%$ del corpo docente. Negli otto anni analizzati, la consistenza dei Pa passa dal $28.6 \%$ al 37.6\%, mentre quella dei Po scende dal $35.2 \%$ al $27.4 \%$. Tale dinamica non è sufficiente a rendere "piramidale" la composizione dei ruoli della docenza, visto che la percentuale dei ricercatori ( $\mathrm{Ru}+\mathrm{Rtd})$, pur con le oscillazioni dovute al Piano Nazionale Associati, rimane pressoché costante nel periodo di tempo analizzato. 
Fig. 2

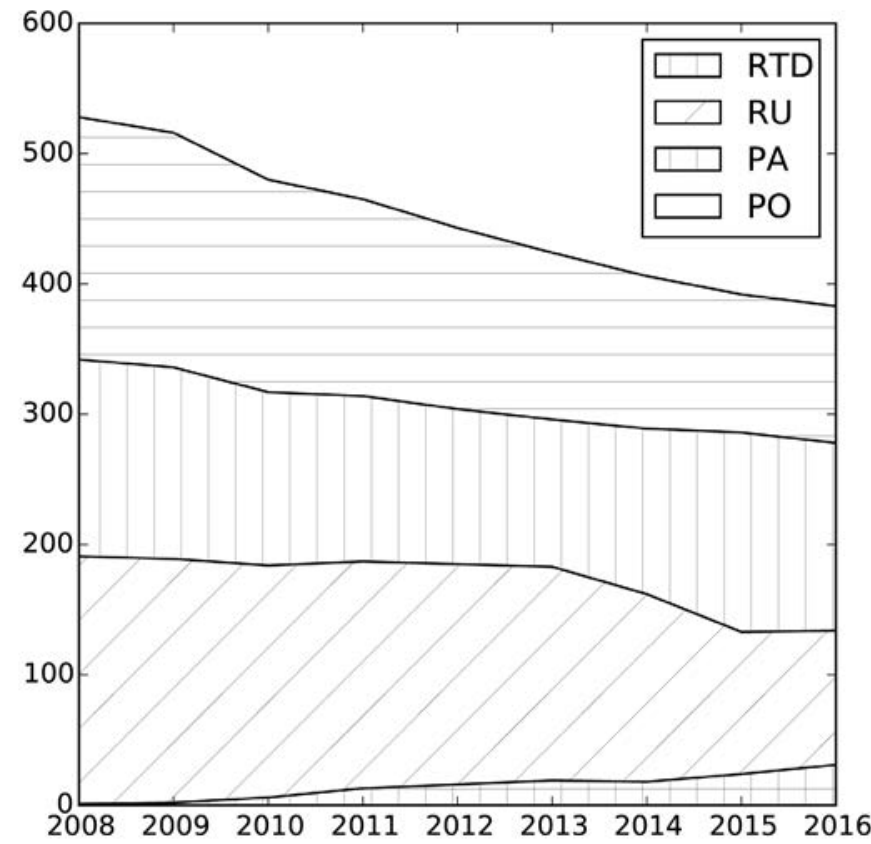

Fig. 3

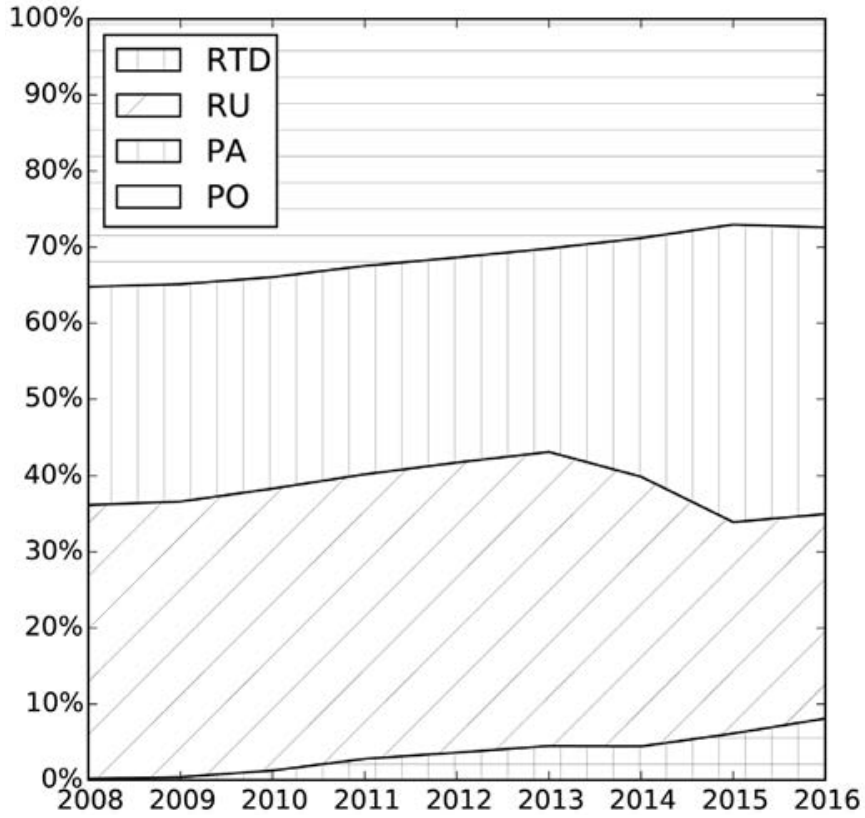

Copyright () FrancoAngeli

Opera pubblicata con Licenza Creative Commons Attribuzione - Non commerciale - Non opere derivate.

Per i termini e le condizioni di utilizzo di questa opera consultare il sito: http://creativecommons.org/. 
Parità di genere. Nonostante la crescita dell'istruzione universitaria femminile negli ultimi decenni, il mondo della ricerca è ancora lontano dalla parità di genere. Negli ultimi anni, tuttavia, il nostro paese sembra aver compiuto alcuni piccoli passi nella giusta direzione. Dal 2008 al 2016, infatti, i docenti di sesso femminile - pur riducendosi da 20.647 a 18.862 - passano dal $34.1 \%$ al 37.3\% sul totale dei docenti. L'Italia, dunque, si avvicina a piccoli passi alla media dell'Unione Europea, dove le donne impiegate nel settore della ricerca universitaria sono circa il $40 \%$ sul totale dei ricercatori.

Nel settore M-sto/04 assistiamo a una dinamica differente rispetto a quella appena descritta. Negli otto anni esaminati, infatti, non assistiamo a una crescita della componente femminile del corpo docente. La percentuale rimane infatti stabile attorno al 33\% su tutti gli anni considerati (Fig. 4).

Fig. 4

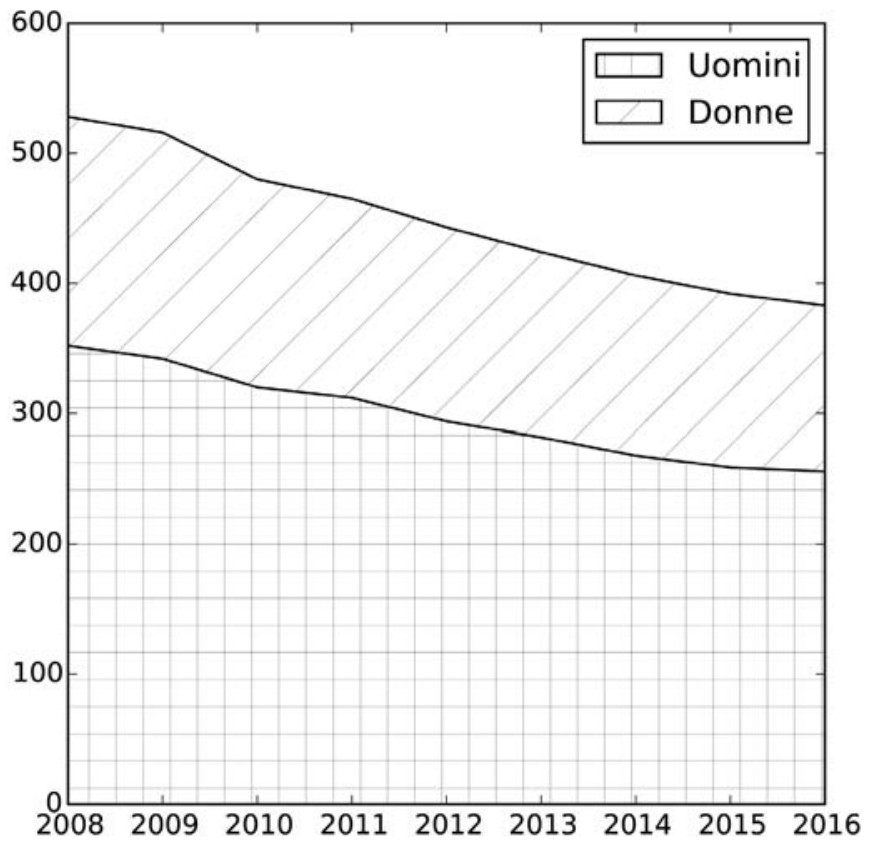

Nel 2008 i docenti di sesso femminile erano 176, pari al 33.3\% del totale; nel 2016 il loro numero si riduce a 127 , pari al $33.1 \%$. La dinamica descritta non è dovuta a un differente numero di uscite tra i due generi (Fig. 5).

La dinamica di genere, non analizzata da Dipper, differenzia dunque il settore della Storia contemporanea da quanto osservato per la globalità delle posizioni da docente universitario nel nostro Paese. 
Fig. 5

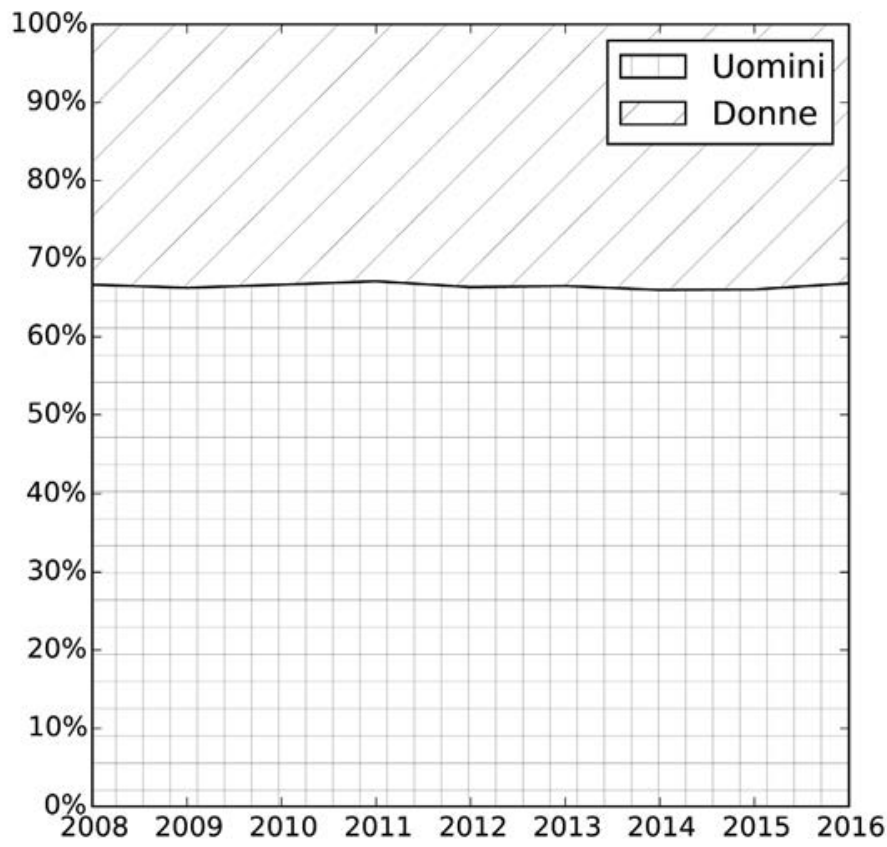

Il corpo docente su base regionale. Per quanto riguarda la distribuzione geografica del corpo docente del settore M-sto/04, nel 2008 il 56.4\% dei docenti risultava concentrato in cinque regioni italiane: Lazio, Toscana, Campania, Emilia-Romagna e Lombardia. Nel Lazio operavano il 16.3\% dei docenti del settore di Storia Contemporanea; la Toscana si collocava in seconda posizione con il $12.7 \%$. Campania, Emilia-Romagna e Lombardia si collocano a breve distanza, con percentuali molto simili $(9.7 \%, 8.9 \%$ e $8.9 \%$ rispettivamente) (Figg. 6 e 7).

Nel 2016 la situazione è molto simile; l'unica eccezione è costituita dalla Lombardia, che ad oggi occupa l'11.2\% dei docenti del settore M-sto/04. Tale espansione è stata conseguita a spese della sola Toscana, che scende dal $12.7 \%$ al 9.9\%. Si delinea un certo processo di "trasferimento" di competenze verso il nord del Paese, dove a farne le spese, nel particolare settore analizzato, sembra essere il centro (Fig. 8).

Dal 2008 al 2011 assistiamo ad una espansione dell'area del Centro, che passa dal $34.7 \%$ al $37 \%$, a spese essenzialmente del Mezzogiorno, che contrae la sua percentuale dal $30.3 \%$ al $28.8 \%$. Negli anni che seguono, invece, vi è una crescita prepotente della percentuale dei docenti impegnati nel Nord del Paese, che sale dal $33.3 \%$ al $36 \%$, mentre le regioni centrali arretrano fino al $32.6 \%$, un calo dunque del $4.4 \%$ (Tab. 1). 
Fig. 6

Docenti universitari settore M-sto/04 per regione - 2008

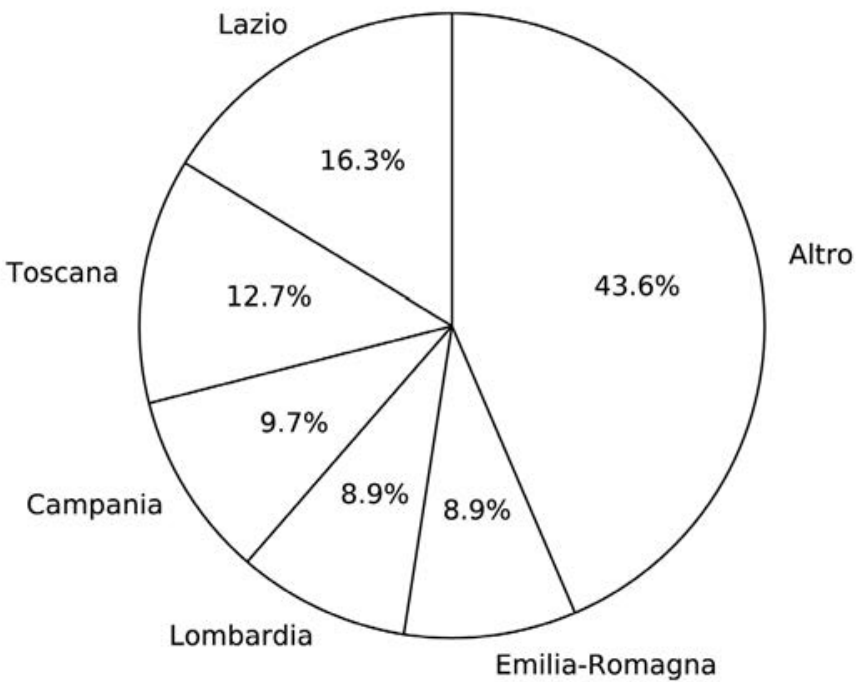

Fig. 7

Docenti universitari settore M-sto/04 per regione - 2016

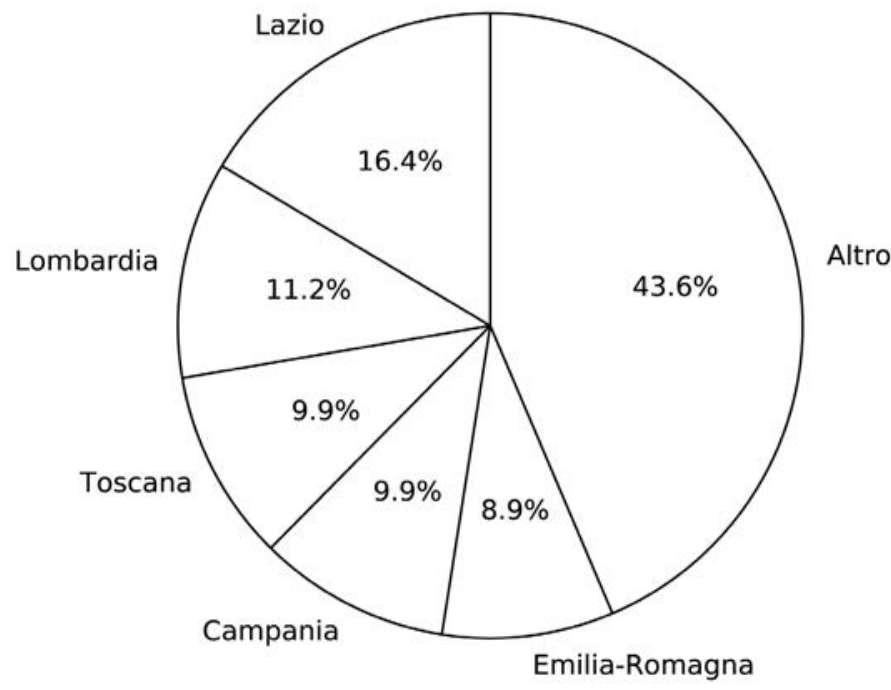

Copyright $\odot$ FrancoAngeli

Opera pubblicata con Licenza Creative Commons Attribuzione - Non commerciale - Non opere derivate.

Per i termini e le condizioni di utilizzo di questa opera consultare il sito: http://creativecommons.org/. 
Fig. 8

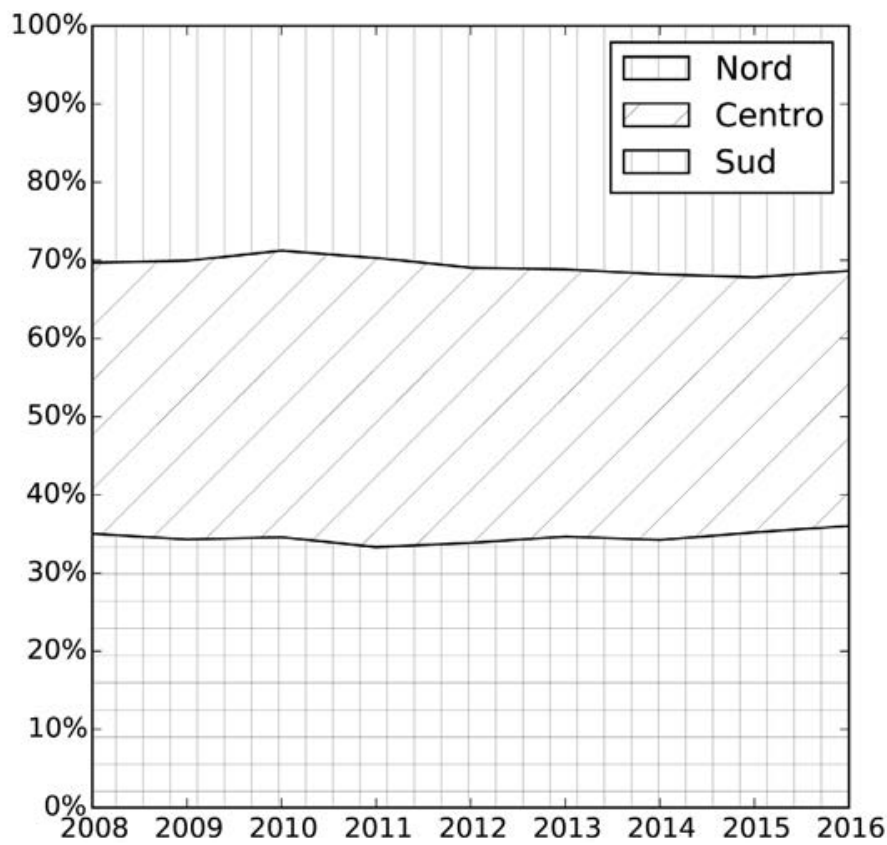

Tab. 1

\begin{tabular}{l|r|r|r}
\hline & 2008 & 2011 & 2016 \\
\hline Nord & $35 \%$ & $33.3 \%$ & $36 \%$ \\
Centro & $34.7 \%$ & $37 \%$ & $32.6 \%$ \\
Sud & $30.3 \%$ & $28.8 \%$ & $31.3 \%$ \\
\hline
\end{tabular}

Reclutamento, progressione di carriera, mobilità. Nel contesto di una generale riduzione delle risorse a disposizione della ricerca, il bilanciamento tra risorse destinate al reclutamento di giovani ricercatori e quelle destinate alle progressioni di carriera è stato oggetto di numerosi dibattiti.

Tab. 2

\begin{tabular}{l|c|c|c|c|c|c|c|c}
\hline & 2009 & 2010 & 2011 & 2012 & 2013 & 2014 & 2015 & 2016 \\
\hline Progressioni & 0 & 8 & 17 & 6 & 2 & 21 & 36 & 13 \\
Reclutamenti & 9 & 16 & 19 & 8 & 6 & 9 & 13 & 12 \\
Totale & 9 & 24 & 36 & 14 & 8 & 30 & 49 & 25 \\
\hline
\end{tabular}

Copyright () FrancoAngeli

Opera pubblicata con Licenza Creative Commons Attribuzione - Non commerciale - Non opere derivate.

Per i termini e le condizioni di utilizzo di questa opera consultare il sito: http://creativecommons.org/. 
La tabella precedente rende evidente il totale blocco del reclutamento nel settore M-sto/04 seguito all'emanazione della legge 240/2010. Esaurita infatti la coda lunga degli ultimi concorsi precedenti la riforma, notiamo come fino al 2014 il reclutamento rimanga quasi totalmente bloccato. Nel 2016 notiamo una ripresa del reclutamento per effetto del piano straordinario $\mathrm{Rtdb}^{27}$. Dalla tabella è anche evidente l'effetto del Piano nazionale associati, che produce più di 50 progressioni di carriera tra 2014 e 2015, con due picchi, rispettivamente nel 2011 e 2015, il primo dovuto al veloce esaurimento dei concorsi pre-Gelmini, il secondo relativo alle risorse straordinarie stanziate (Fig. 9).

Fig. 9

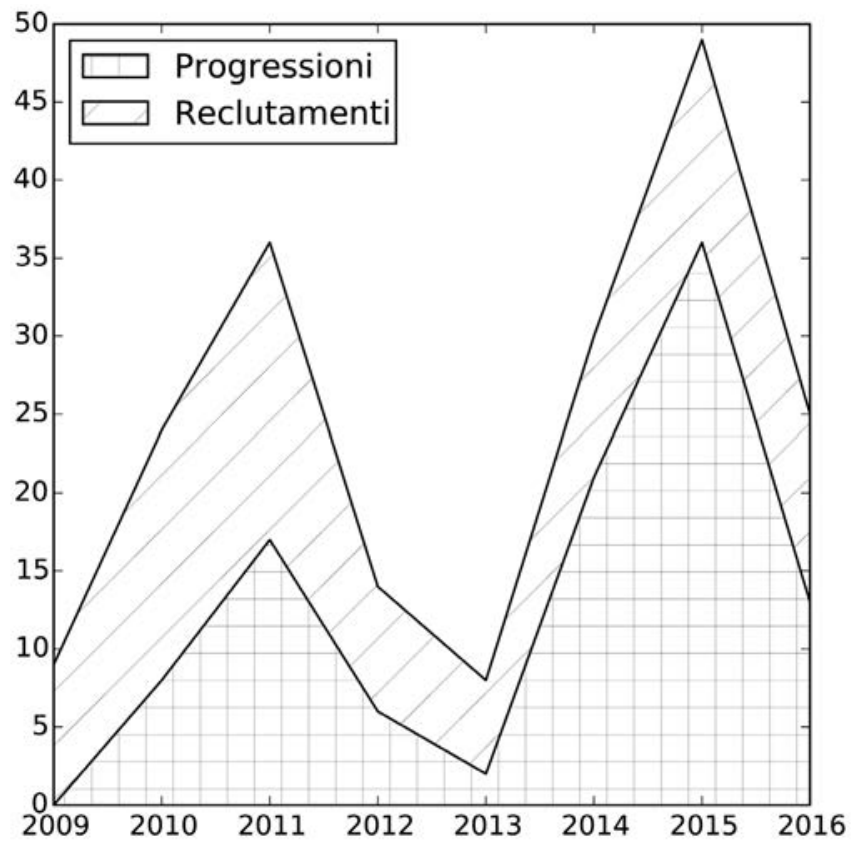

In valori assoluti, dal 2009 al 2011 e in regime concorsuale pre-Gelmini, i reclutamenti sono in misura nettamente maggiore rispetto alle progressioni di carriera; la percentuale si inverte negli ultimi tre anni, dove il rapporto tra progressioni di carriera e reclutamento risulta nettamente squilibrato a favore delle progressioni (Fig. 10).

${ }^{27}$ Si veda il Dm 18 febbraio 2016 n. 78, "Piano Straordinario 2016 per il reclutamento di ricercatori di cui all'articolo 24, comma 3, lettera b) della legge 240/2010, http://attiministeriali. miur.it/anno-2016/febbraio/dm-18022016.aspx. 
Fig. 10

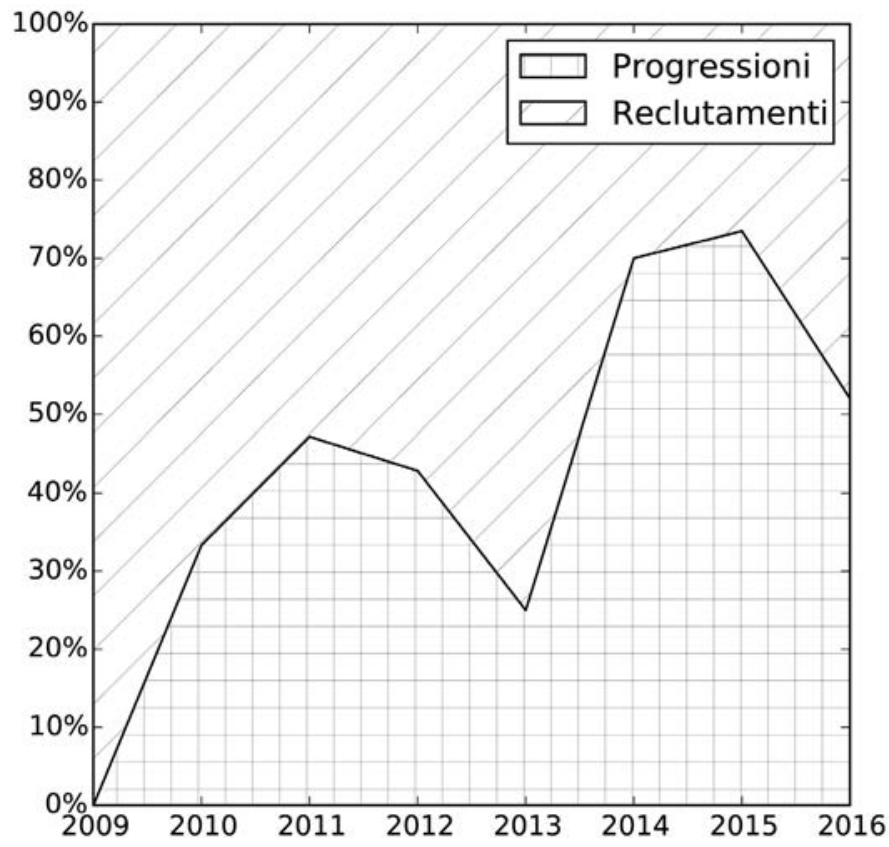

Le dinamiche relative al reclutamento per genere ad area geografica risultano coerenti con i risultati illustrati in precedenza.

Tab. 3

\begin{tabular}{l|l|l|l|l}
\hline Anno & $\begin{array}{l}\text { Ateneo } \\
\text { di provenienza }\end{array}$ & Ruolo & $\begin{array}{l}\text { Ateneo } \\
\text { di destinazione }\end{array}$ & Ruolo \\
\hline 2009 & $\begin{array}{l}\text { S. Raffaele } \\
\text { Milano }\end{array}$ & Ordinario & $\begin{array}{l}\text { Sum - Istituto } \\
\text { Italiano di Scienze } \\
\text { umane Firenze }\end{array}$ & Ordinario \\
\hline 2011 & $\begin{array}{l}\text { Univ. Telematica } \\
\text { "E-Campus" }\end{array}$ & Rtd & $\begin{array}{l}\text { Campania - } \\
\text { "L. Vanvitelli" }\end{array}$ & Ricercatore \\
\hline 2012 & Teramo & Associato & $\begin{array}{l}\text { Roma } \\
\text { "La Sapienza" }\end{array}$ & Associato \\
\hline 2015 & Teramo & Associato & Milano & Associato \\
\hline 2015 & Genova & Ricercatore & $\begin{array}{l}\text { "Ca' Foscari” } \\
\text { Venezia }\end{array}$ & Ricercatore \\
\hline 2016 & Bergamo & Ricercatore & Roma Tre & Associato \\
\hline 2016 & Salerno & Ricercatore & Basilicata & Ricercatore \\
\hline
\end{tabular}

Copyright () FrancoAngeli

Opera pubblicata con Licenza Creative Commons Attribuzione - Non commerciale - Non opere derivate.

Per i termini e le condizioni di utilizzo di questa opera consultare il sito: http://creativecommons.org/. 
Per quanto riguarda la mobilità tra atenei, nel settore M-sto/04 essa si dimostra un fenomeno del tutto residuale. Abbiamo infatti solo 7 casi di mobilità tra atenei, e due di essi corrispondono a progressioni di carriera; la stragrande maggioranza dei casi di mobilità, inoltre, riguarda professori associati e ricercatori (un solo caso, nel 2009, è relativo ad un ordinario). Nel complesso, nell'anno 2016, solo lo $0.5 \%$ del personale docente si è trasferito da un ateneo ad un altro (Tab. 3).

In conclusione, ci sembra che le sollecitazioni di Christof Dipper vadano tenute in debita considerazione e contribuiscano a definire, da uno sguardo esterno, i contorni di alcuni caratteri di fondo degli studi storico-contemporaneistici in Italia. Tuttavia, esse fanno forse eccessivo affidamento su una lettura in chiave "culturalista" che, a nostro modo di vedere, andrebbe confrontata con una più attenta analisi delle conseguenze imposte dai più recenti provvedimenti di riforma. Ci è infine parso di cogliere un giudizio negativo sull'esito dell'università di massa in Italia. Per questo motivo abbiamo insistito sul rapporto di funzionalità che lega un decennio di riforme alla competizione economica internazionale. Crediamo che un dibattito approfondito in questo senso non si sia ancora pienamente sviluppato. 